\title{
1 Electrophysiological identification of daily rhythms in the prefrontal cortex
}

2 Brandon L. Roberts ${ }^{1}$, Jiexin Wang ${ }^{1}$ and Ilia N. Karatsoreos ${ }^{1}$

$4{ }^{1}$ Neuroscience and Behavior Program, and Department of Psychological and Brain Sciences,

5 University of Massachusetts Amherst, Amherst, MA 01003, USA

7 Running Head: Daily rhythms in PFC

9 Correspondence should be sent to:

10 Ilia N. Karatsoreos, Ph.D.

11 Department of Psychological and Brain Sciences

12 University of Massachusetts Amherst

13 Tobin Hall, 135 Hicks Way

14 Amherst, MA 01003

15 E-mail: ikaratsoreos@umass.edu

16

17 Word count: Abstract (199), Introduction (756), Results (1,436), Discussion $(2,237)$, Methods $(1,027)$

18 Figures: (6)

19 Figure supplements: (2)

20 Figure data files: (7) 


\section{Abstract}

22 Circadian rhythms are ubiquitous in biology, from the molecular to behavioral levels. There is growing

23 interest in understanding the functional implications of circadian oscillations in different cells and

24 systems, including the brain. The prefrontal cortex (PFC) is heavily involved in myriad processes,

25 including working memory, cognition, stress responses, and fear associated behaviors. Many PFC

26 associated behaviors are time-of-day dependent, yet how time-of-day impacts the basic function of

27 neurons in the PFC is not known. Here we use patch-clamp electrophysiology to record from layer $2 / 3$

28 pyramidal neurons in the prelimbic ( $\mathrm{pl}$ ) PFC of male and female C57BL/6J mice at 4 separate bins of

29 zeitgeber time (ZT): 0-4, 6-10, 12-16, and 18-22. We measured changes in membrane properties,

30 inhibitory and excitatory inputs, ion channel function, and action potential kinetics. We demonstrate that

31 the activity of pIPFC neurons, their inhibitory inputs, and action potential dynamics are regulated by

32 time-of-day. Further, we show that in males postsynaptic $\mathrm{K}^{+}$channels play a central role in mediating

33 these rhythms, suggesting the potential for an intrinsic gating mechanism mediating information

34 throughput. These key discoveries in PFC physiology demonstrate the importance of understanding

35 how daily rhythms contribute to the mechanisms underlying the basic functions of PFC circuitry. 


\section{Introduction}

Rhythms in life are found at many different time scales in nearly all phyla. From annual rhythms in hibernation and reproduction, to daily rhythms in sleep-wake cycles, to ultradian rhythms such as variations in heart rate, to rhythms in coordinated brain activity (Helm et al. 2013; Körtner and Geiser 2000; Yaniv and Lakatta 2015; M. H. Hastings, Reddy, and Maywood 2003). They are also present at nearly all levels of organization, from the behavior of groups of organisms to gene and protein expression at the cellular level (Jagannath et al. 2017; Landgraf et al. 2016; J. W. Hastings 2007). Given their ubiquity in nature and involvement in countless biological processes, understanding the functional significance of these rhythms is critical. However, while major strides have been made in understanding how these rhythms impact cellular function in the suprachiasmatic nucleus (SCN), as well as in some peripheral organs such as the liver, there remains a paucity of information about the functional impact of circadian clocks in other brain regions, beyond identifying that circadian rhythms are present (Abe et al. 2002; Albrecht and Stork 2017; Sato et al. 2020; Weaver 1998). This is a major gap in our knowledge, considering that circadian rhythms in behaviors are well documented, and shown to be critical in both health and disease.

The PFC serves as a critical component in cognition, emotional systems involved in fear learning and extinction, stress responses, and learning and memory, all of which are impacted by daily rhythms (Woodruff et al. 2018; McCarthy and Welsh 2012; Popoli et al. 2012; Sotres-Bayon, Cain, and LeDoux 2006; Miller and Cohen 2001). In addition, clock gene expression has been documented in the prefrontal cortex (PFC) (Chun et al. 2015). The prelimbic area (pl) of the PFC is divided into six distinct layers, each with distinct inputs and projections. Specifically, layer 2/3 plays a major role in working memory and behavioral plasticity and is involved in stress and depressive behaviors (Yuen et al. 2009; Zaitsev et al. 2012; Radnikow and Feldmeyer 2018; Moorman et al. 2015). The PFC is comprised of a

61 information to other brain regions that are under clear circadian control, such as the amygdala and 62 hippocampus, and inhibitory interneurons, such as the parvalbumin $(P V+)$ and neuropeptide Y (NPY) 63 containing neurons (Kawaguchi and Kubota 1997; Radnikow and Feldmeyer 2018; Vertes 2006; Saffari 
et al. 2016). On whole, the function, synaptic inputs and intrinsic physiological characterizations of these neurons are heterogeneous throughout the PFC.

pIPFC pyramidal neurons receive excitatory glutamatergic and inhibitory GABAergic presynaptic input, with findings suggesting these are differentially regulated in male and female mice (Popoli et al. 2012; Saffari et al. 2016; Andrade et al. 2012; de Velasco et al. 2015; Pena-Bravo et al. 2019). Excitatory inputs onto these neurons are plastic and environmental factors such as stress, learning and memory, can lead to long-lasting potentiation of glutamatergic inputs onto these neurons through increased NMDAR and AMPAR mediated currents (Yuen et al. 2009; Laroche, Jay, and Thierry 1990).

Over activation of pIPFC neurons is detrimental to normal behavioral function, and inhibitory inputs, which arise from the numerous inhibitory interneurons throughout the PFC, serve to mitigate the excitability of PFC neurons (Ferguson and Gao 2018). The majority of PFC pyramidal neurons are intrinsically quiescent at rest and regulate information throughput via a wide array of ion channels, including cyclic-nucleotide-gate non-selective cation $(\mathrm{HCN})$ channels, and calcium $\left(\mathrm{Ca}^{2+}\right)$ and potassium $\left(\mathrm{K}^{+}\right)$channels known to mediate postsynaptic throughput of excitatory and inhibitory currents (Kalmbach and Brager 2020; Zaitsev et al. 2012; Deng et al. 2019; Workman et al. 2015). In the SCN, changes in sodium $\left(\mathrm{Na}^{+}\right), \mathrm{K}^{+}$, and $\mathrm{Ca}^{2+}$ ion channel function mediate daily rhythms in the spontaneous activity, and action potential dynamics of neurons (Bano-Otalora et al. 2021). How these channels might impact daily rhythms in PFC function and the gating of information throughput is unknown. previously documented effects of circadian desynchronization on PFC structure (Karatsoreos et al. 2011), here we rigorously tested how time-of-day alters a wide range of neurophysiological properties in pIPFC pyramidal neurons. The data presented here thoroughly demonstrate that time-of-day clearly pyramidal neurons.

inputs fluctuate throughout the day in a sex dependent manner. Lastly, we identify that $\mathrm{K}^{+}$channels may serve, in part, as a mechanism to regulate daily changes in information throughput in pIPFC 
bioRxiv preprint doi: https://doi.org/10.1101/2022.01.27.478010; this version posted January 28, 2022. The copyright holder for this preprint (which was not certified by peer review) is the author/funder, who has granted bioRxiv a license to display the preprint in perpetuity. It is made available under aCC-BY-NC-ND 4.0 International license.

\section{Results}

92 Resting membrane potential of prelimbic layer 2/3 pyramidal neurons is rhythmic in male mice.

The regional and cell specific heterogeneity in electrophysiological properties of PFC pyramidal neurons has been described in multiple species (Zaitsev et al. 2012; van Aerde and Feldmeyer 2015; Piette et al. 2021). Layer 2/3 pyramidal neurons of the pIPFC were identified visually by anatomical location (Figure 1A; left). Pyramidal neurons were identified by shape and lucifer yellow (LY; 0.2\%) was added to the patch pipette for confirmation of an apical dendrite (Figure 1A; right). pyramidal neurons, we performed whole-cell patch clamp techniques and measured RMP, membrane capacitance $(\mathrm{Cm})$, and membrane resistance $(\mathrm{Rm})$ at ZT bins: 0-4, 6-10, 12-16, and 18-22 in male and female mice (Figure 1B-E). For RMP there was a main effect of time (no effect of sex) and

A

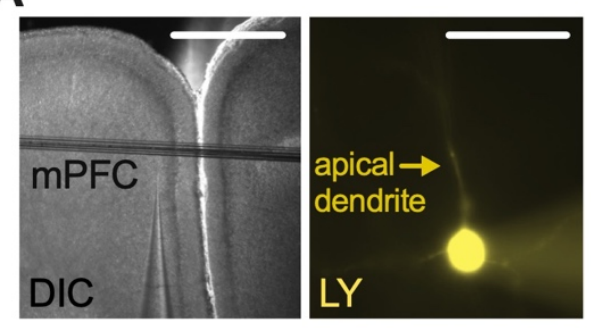

C

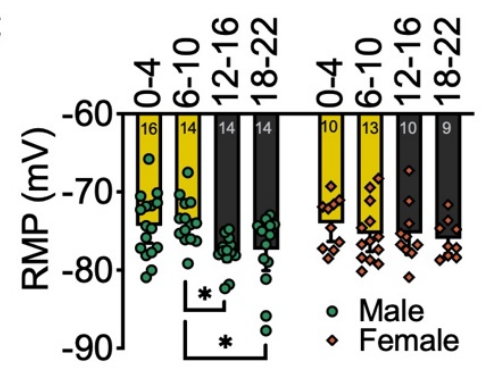

B

\section{$-70 \mathrm{mV}$}

ZT 0-4

ZT 6-10

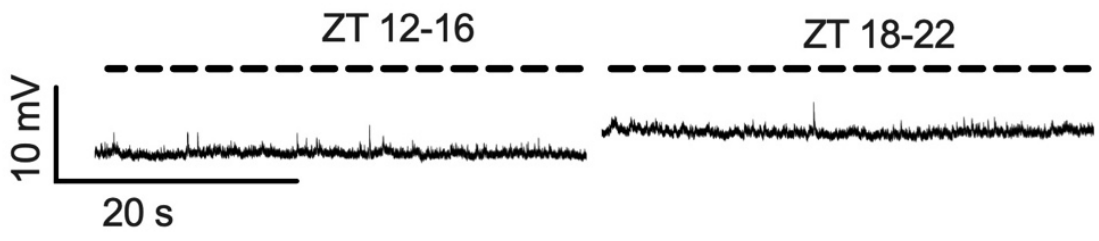

E

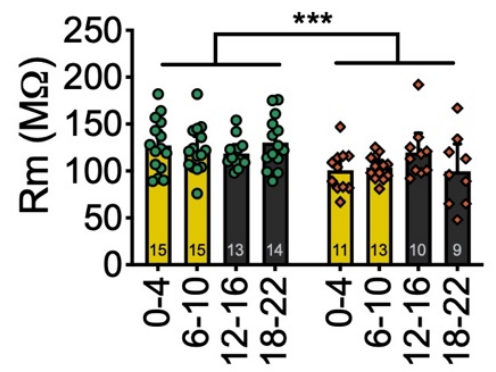

ZT

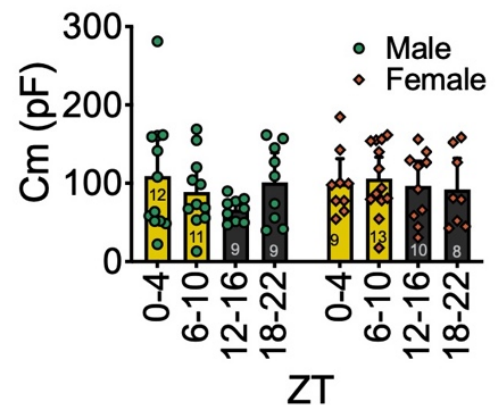


Figure 1. Time-of-day changes in membrane potential of layer $2 / 3$ mPFC pyramidal neurons.

(A) Image of mPFC slice (left; scale $1 \mathrm{mM}$ ) and layer 2/3 pyramidal neuron backfilled with lucifer yellow (LY, right; scale $10 \mu \mathrm{M})$. (B) Representative traces of current clamp recordings from male mice at each ZT bin. (C) Mean and individual data points for membrane potential (RMP) at ZT04, 6-10, 12-16, and 18-22 in male (bluish green circles) and female (vermillian diamonds) mice (D) Mean membrane capacitance $(\mathrm{Cm})$ and $(\mathrm{E})$ resistance $(\mathrm{Rm})$ binned by ZT. Error bars represent $\pm 95 \% \mathrm{Cl}$. N-values for number of cells inset on bars. Two-way ANOVA for main effects and interaction with a within group Tukey post-hoc analysis for ZT bin, ${ }^{*} p<0.05$, ${ }^{* * *} p<$ 0.001. Exact $\mathrm{p}$-values, mouse $\mathrm{N}$-values, and analysis in Figure 1 - source data 1.

109

\section{sEPSC activity on pIPFC pyramidal neurons is time-of-day dependent}

Glutamatergic pyramidal neurons are the predominant cell-type in the pIPFC, project to extraPFC cortical, subcortical and limbic regions, and interconnect within the PFC (Le Merre, ÄhrlundRichter, and Carlén 2021). We hypothesized that basal excitatory glutamatergic release contributes to daily changes in RMP and predicted that the number and/or strength of excitatory inputs are highest during the light period, when pIPFC pyramidal neurons are depolarized (Figure 1B, C). To test whether time-of-day alters excitatory inputs we used the whole-cell voltage-clamp configuration $\left(\mathrm{V}_{\mathrm{H}}=-70 \mathrm{mV}\right)$ to record sEPSCs in pIPFC pyramidal neurons from male and female mice at ZTO-4, 6-10, 12-16, and 1822 (Figure 2A-E). There was a main effect of ZT time on sEPSC frequency, but not amplitude, and post-hoc analysis demonstrated that in male mice the frequency of excitatory inputs was increased during the dark period, when RMP is hyperpolarized, an effect counter to our hypothesis (Figure 1B and 2A-C). Further, we observed clear sex differences on both sEPSC frequency and amplitude (Figure 2D, E). These data suggest that time-of-day impacts the number of excitatory inputs (frequency), but not their strength (amplitude) in male and female mice. 


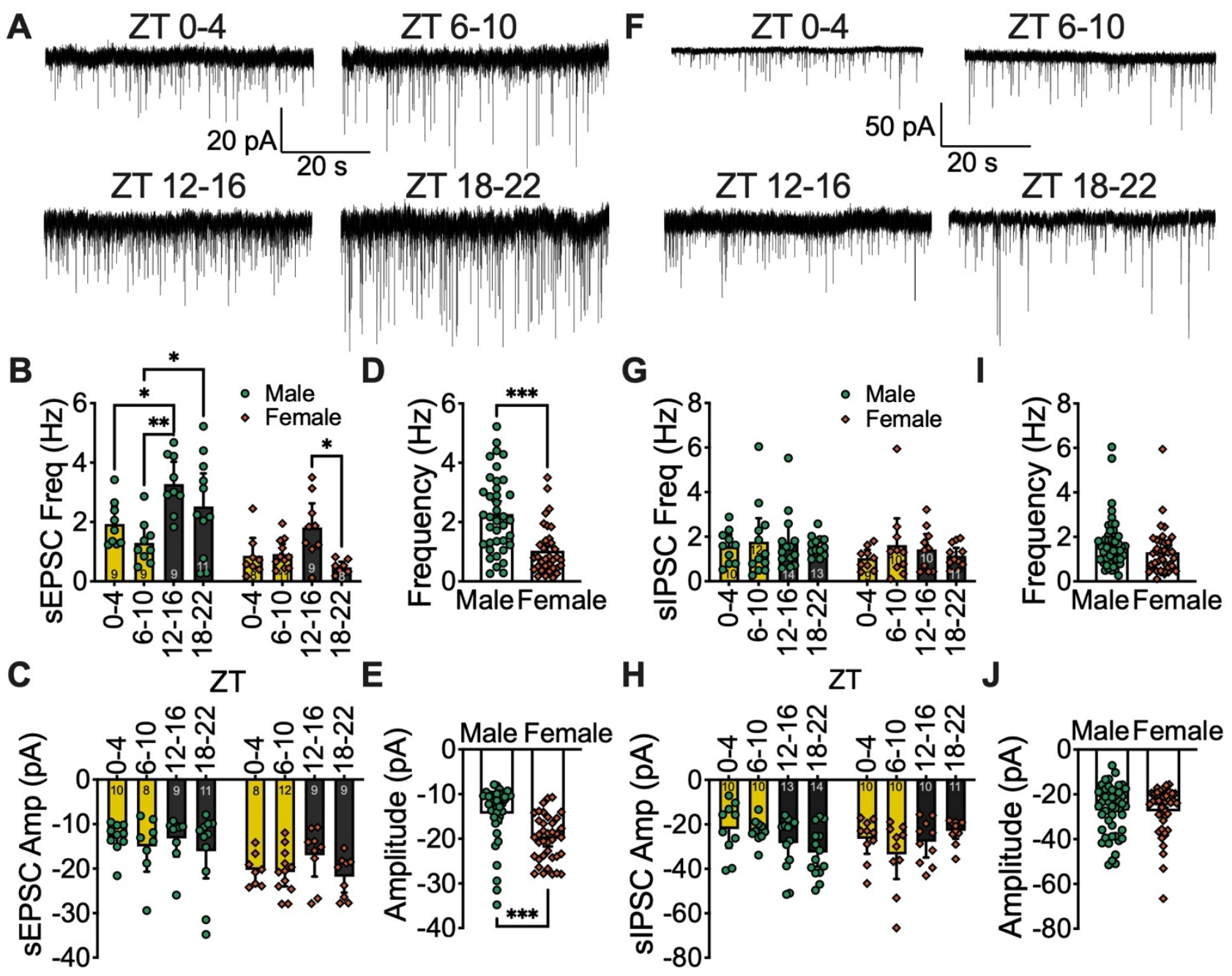

Figure 2. Excitatory synaptic inputs onto pIPFC pyramidal neurons are time-of-day dependent and differ by sex. (A) Representative traces of sEPSC voltage clamp recordings from male mice at each ZT bin. (B, D) Mean and individual data points for sEPSC frequency and (C, E) amplitude at ZT0-4, 6-10, 12-16, and 18-22 or combined (respectively) in male (bluish green circles) and female (vermillian diamonds). (F) Representative traces of sIPSC voltage clamp recordings from male mice at each ZT bin. (G, I) Mean and individual data points for sIPSC frequency and (H, J) amplitude at ZT0-4, 6-10, 12-16, and 18-22 or combined (respectively) in male and female. Error bars represent \pm $95 \% \mathrm{Cl}$. N-values for number of cells inset on bars. Two-way ANOVA for main effects (including $\boldsymbol{D}, \boldsymbol{E}$, $\boldsymbol{I}$ and $\boldsymbol{J}$ ) and interaction, with a within group Tukey post-hoc analysis for ZT bin, ${ }^{*} p<0.05,{ }^{* *} p<0.01$, ${ }^{* * *} p<0.001$. Exact $p$-values and analysis in Figure 2 - source data 1. 
127 Time-of-day does not alter sIPSCs in male or female mice

sEPSC frequency and amplitude (Figure 2A-E) in the pIPFC suggest that excitatory synaptic inputs are not the primary driver of the daily changes in RMP illustrated in Figure 1 and required further investigation to identify the mechanism underlying time-of-day changes in resting state. To investigate

132 the contrast between daily changes in RMP and excitatory inputs, we predicted that if synaptic signaling

133 is a primary mediator of time-of-day changes in RMP then inhibitory inputs should be robust and

134 highest during the dark period ZT bins. GABAergic interneurons make up a small portion of PFC

135 neurons, but are highly involved in the regulation of pyramidal neurons and relay information between

136 different regions within the PFC (Saffari et al. 2016). To determine if inhibitory inputs contribute to the

137 diurnal tone of pyramidal neurons, we recorded (s) inhibitory postsynaptic currents (IPSCs; $\mathrm{V}_{\text {Hold }}=$ -

$13870 \mathrm{mV}$ ) using a cesium chloride internal solution at ZT 0-4, 6-10, 12-16, and 18-22 (Figure 2F-H). We

139 did not observe a significant difference in sIPSC frequency or amplitude in male or female mice (Figure

140 2F-J), but noted a trend in increased amplitude during the dark period in male mice (Figure 2 F, H).

141 Overall, these data do not support the notion that spontaneous synaptic inputs are the primary regulator 142 of daily rhythms in neuronal resting state in pIPFC pyramidal neurons, suggesting that a postsynaptic 143 mechanism may regulate daily rhythms in these neurons.

mIPSCs onto pIPFC pyramidal neurons are time-of-day dependent

Our central goal was to identify the mechanism(s) by which daily rhythms impact information

147 throughput of pIPFC pyramidal neurons. Given that we did not observe daily changes of RMP in female 148 mice, and there was a main effect of sex on membrane resistance (Figure 1C, E), sEPSC frequency, 149 and amplitude (Figure 2D, E) - all without an interaction between sex and ZT bin - we proceeded with 150 our mechanistic investigation by separating male and females into independent groups.

151 Although we did not observe an effect of ZT bin on sIPSC frequency or amplitude in male mice, 152 when ZT bins were combined into light period and dark period, we uncovered a significant impact of the 153 LD cycle on sIPSC amplitude (Figure 3B). To further investigate whether time-of-day impacts the 
154 presynaptic or postsynaptic components of inhibitory inputs, we measured mIPSCs by bath applying

155 the voltage-gated sodium channel blocker tetrodotoxin, which isolates the synapse from upstream

156 activity by inhibiting action potential firing. We observed no effect on mIPSC frequency, which is

157 typically associated with presynaptic neurotransmitter release, in male mice (Figure 3A, C). Although

158 we did not observe changes in decay tau or holding current, the time-of-day effect on IPSC amplitude

159 persisted in this configuration, and this increase in amplitude of inhibitory inputs is consistent with the

160 hyperpolarized RMP we observed during the dark period in male mice (Figure 1C and 3A, D-F).

161 Notably, in female mice there was no LD effect on sIPSC amplitude (Figure 3H), but we did observe an

162 increase in mIPSC frequency and amplitude during the latter part of the light period (ZT 6-10; Figure

163 3G, I-J) although there was no effect on other potential postsynaptic measures, such as decay tau and

164 holding current (Figure 3K, L). 
A ZT 0-4 ZT 6-10 Male ZT 12-16 ZT 18-22

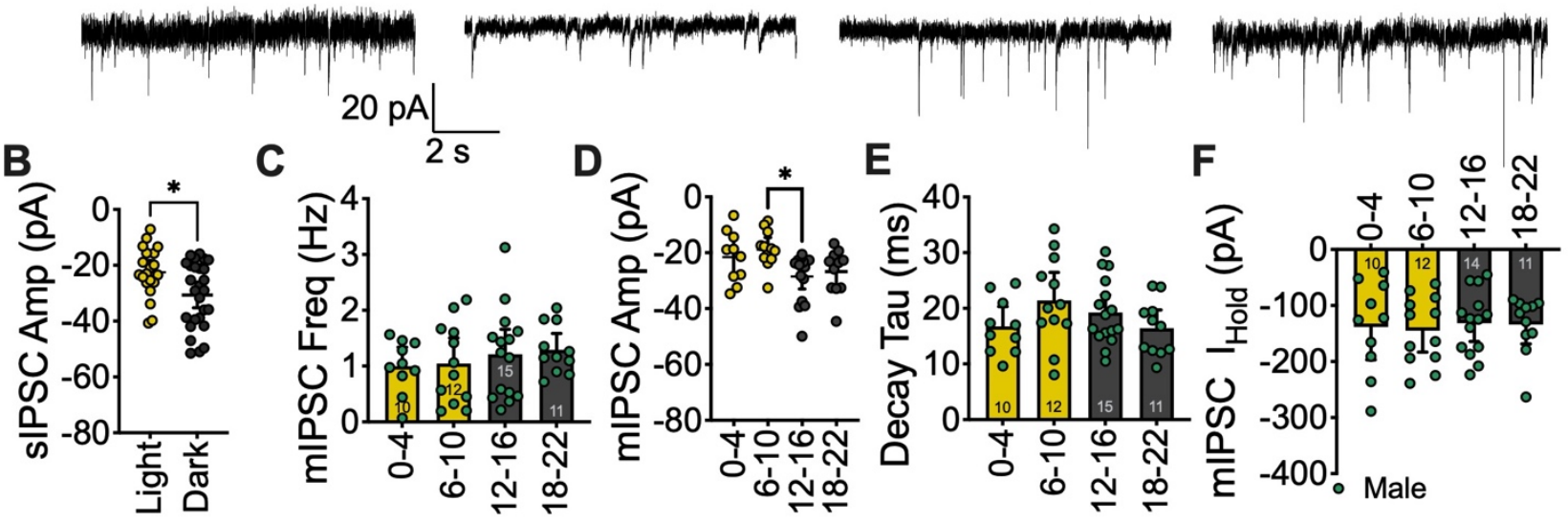

G
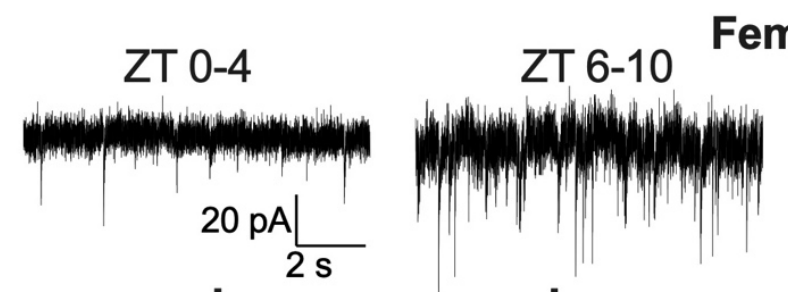

Female
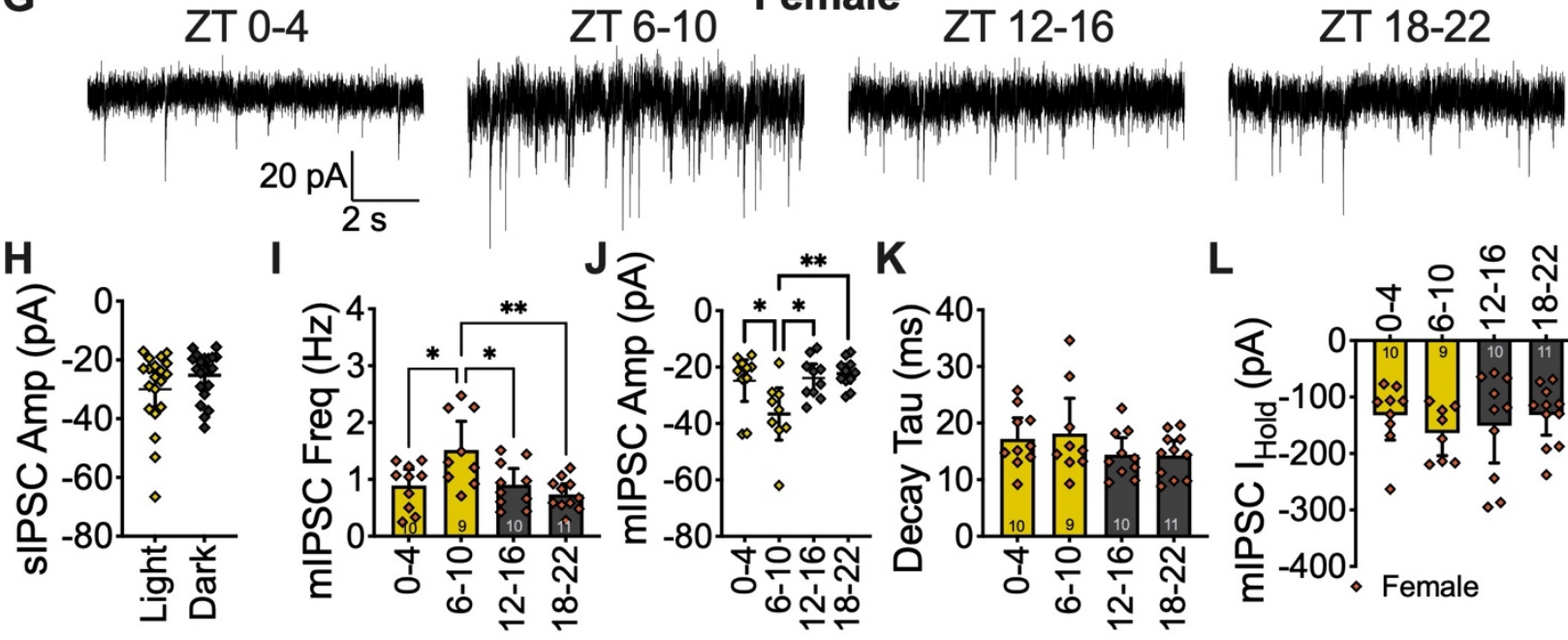

Figure 3. Strength of inhibitory synaptic inputs onto pIPFC pyramidal neurons are time-of-day dependent in male and female mice. (A) Representative traces of mIPSC voltage clamp recordings from male and (G) female mice at each ZT bin. (B, H) Mean sIPSC amplitude in LD cycle (unpaired student t-test; yellow fill = light period, charcoal fill = dark period) and (C, I) mIPSC frequency, (D, J) amplitude, $(E, K)$ decay tau, and $(F, L)$ holding current (l Hold $_{\text {d }}$ at ZTO-4, 6-10, 12-16, and 18-22 in male (circles) and female (diamonds) mice. Error bars represent $\pm 95 \% \mathrm{Cl}$. One-way ANOVA with Tukey post-hoc analysis for ZT bin, ${ }^{*} p<0.05,{ }^{* *} p<0.01$. N-values for number of cells inset on bars, exact p-values, mouse $\mathrm{N}$-values, and analysis in Figure 3 - source data 1. 
167 Time-of-day impact on current voltage relationship in pIPFC pyramidal neurons of female mice

Although mIPSCs were enhanced during the latter portion of the light period (ZT6-10) in female

169 mice, we had not observed any convincing evidence of cell endogenous daily rhythms in the physiology

170 of pIPFC pyramidal neurons in these mice. To investigate any potential daily changes in postsynaptic

171 properties we used a potassium $\left(\mathrm{K}^{+}\right)$gluconate internal solution and measured the current-voltage $(\mathrm{I}-\mathrm{V})$

172 relationship in these neurons by performing a voltage-step inactivation protocol in which neurons held

173 at $-70 \mathrm{mV}$ were depolarized to $30 \mathrm{mV}$ and hyperpolarized in $10 \mathrm{mV}$ steps to a final MP of $-120 \mathrm{mV}$ (Figure

174 4A, B). We analyzed the delayed steady-state current density (current density $\left.=\left(\mathrm{I}_{\text {Total }}\right) /(\mathrm{Cm})\right)$ during the

175 hyperpolarized (from $-120 \mathrm{mV}$ to $-70 \mathrm{mV}(K 1)$; Figure $4 \mathrm{~A}-\mathrm{C}$ ) and depolarized state (0 $\mathrm{mV}$ to $30 \mathrm{mV}(K 2)$;

176 Figure 4A, B, E), as well as daily changes in normalized conductance $(\mathrm{g})$ calculated as the slope of the

$177 \mathrm{~K} 1$ and $\mathrm{K} 2$ steady-state current normalized to cell capacitance: $g_{\text {Normalized }}=\left(\left(\mathrm{I}_{\mathrm{VH} 2}-\mathrm{I}_{\mathrm{VH} 1}\right) /\left(\mathrm{V}_{\mathrm{H}} 2-\right.\right.$

$\left.\left.178 \mathrm{~V}_{\mathrm{H}} 1\right)\right) /(\mathrm{Cm})$, at different ZT bins (Figure 4D, F). We observed a main time-of-day effect on current

179 density and normalized conductance for the K1 hyperpolarized voltage steps, although there was no

180 main effect at the K2 depolarized voltage steps (Figure 4B-F). Together, these data demonstrate that

181 female mice do display daily rhythms in postsynaptic membrane properties, but they are not robust

182 enough to alter resting state (Figure 1C-E). 
A

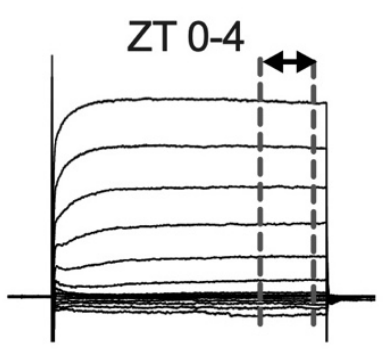

ZT 12-16

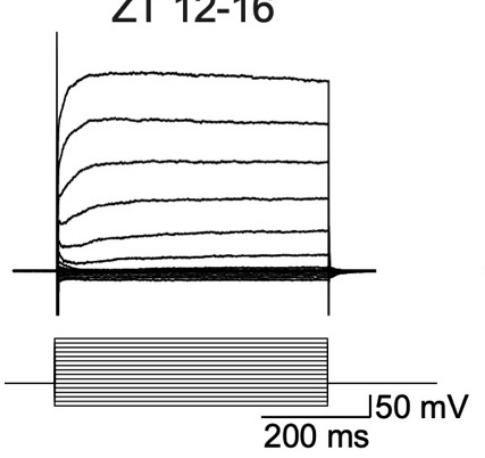

B

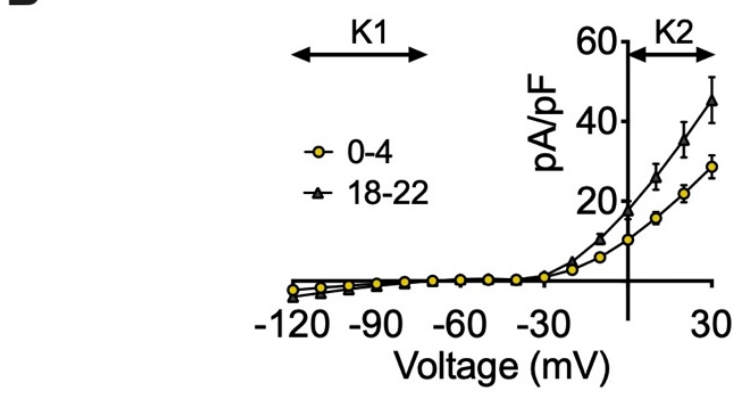

ZT 6-10

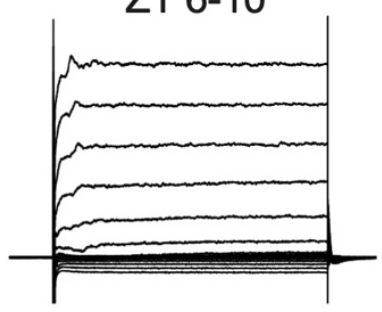

ZT 18-22

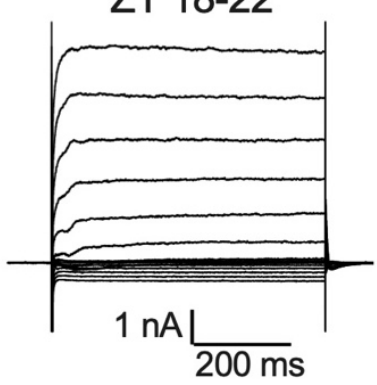

C

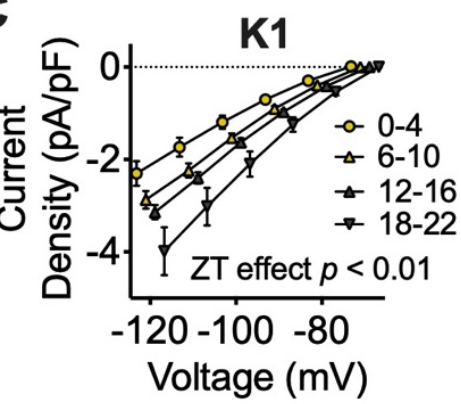

D
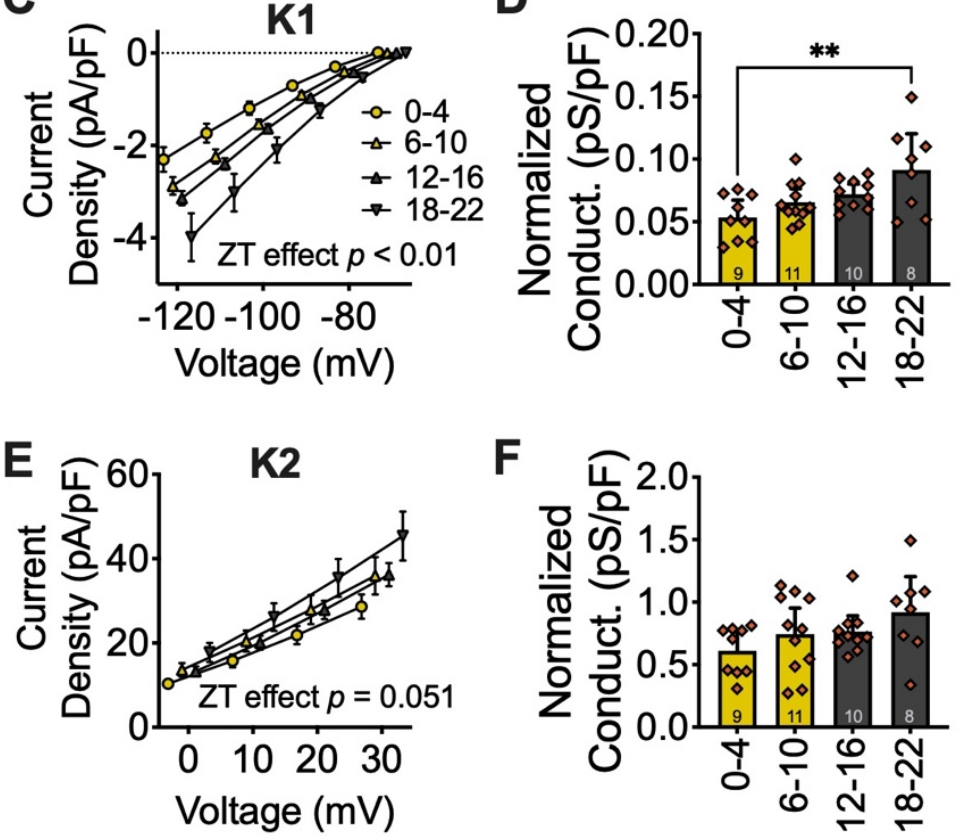
Figure 4. Time-of-day impact on membrane conductance and currents in female mice. (A) Currentvoltage trace (top; dashed line represents steady state current averaged for analysis) after voltagestep protocol (bottom). (B) Averaged I-V voltage-step relationship from -120mV to 30mV for ZT0-4 and 18-22 (normalized to cell capacitance) with a $\mathrm{K}^{+}$internal solution. (C, E) Current density and (D, F) conductance of K1 and K2 currents (respectively) at ZT0-4, 6-10, 12-16, and 18-22 in female mice. K1 and K2 represent hyperpolarized and depolarized currents (respectively). Error bars represent $\pm 95 \% \mathrm{Cl}$. Two-way $(C, E)$ or One-way $(D, F)$ ANOVA with Tukey post-hoc analysis for ZT bin. ${ }^{* *} p<0.01$. N-values for number of cells inset on bars, exact $p$-values and analysis in Figure 4 source data 1.

Time-of-day impact on current voltage relationship in pIPFC pyramidal neurons of male mice Given that synaptic inputs are rhythmic in male and female mice, but only male mice display daily rhythms in RMP, we hypothesized that changes in postsynaptic ionic currents, as measured by the I-V relationship, may play a role in setting the functional tone of pIPFC pyramidal neurons in male mice. As in Figure 4, we analyzed the I-V relationship by running a voltage-step protocol (Figure 5A, B). In male mice the I-V relationship demonstrated a clear inward rectifying current at lower holding voltages and a delayed rectifying current at depolarizing voltages, resulting in a larger current density early in the dark period (ZT12-16) in both the hyperpolarized (Figure 5A-C) and depolarized state

194 (Figure 5A, B, D). This effect translated into higher normalized cell conductance late in the light period 195 and early in the dark period (Figure 5I, J).

Since ionic conductance was highest at ZT12-16, the same ZT bin that RMP was most 197 hyperpolarized, we predicted that this increase involved ion channel activity that results in a net 198 negative current. Further, in the hyperpolarized state, the current density at each ZT began to converge 199 near our calculated reversal potential for $\mathrm{K}^{+}$. To determine if daily changes in current density and 200 normalized cell conductance was dependent on $\mathrm{K}^{+}$channel activity, we utilized a $\mathrm{K}^{+}$free $\mathrm{Cs}^{+}$-based 201 internal recording solution to block outward $\mathrm{K}^{+}$currents. This preparation completely abolished the time202 of-day effect on current density at lower holding voltages, but not in the depolarized state (Figure 5E- 
203 H). Further, the time-of-day effect on normalized cell conductance was blocked when calculated at the 204 presented voltages with a main effect of $\mathrm{Cs}^{+}$on overall conductance in the depolarized state (Figure $205 \mathbf{5 I , J ) . ~ O f ~ p a r t i c u l a r ~ n o t e , ~ b l o c k a d e ~ o f ~ o u t w a r d ~} \mathrm{K}^{+}$currents via internal $\mathrm{Cs}^{+}$appeared to have little effect 206 at ZT0-4, suggesting minimal $\mathrm{K}^{+}$channel activity at this ZT bin (Figure 5C,G). Together, these data 207 demonstrate that ion channel activity is time-of-day dependent in pIPFC pyramidal neurons and $\mathrm{K}^{+}$ 208 channels contribute to daily rhythms in their cellular conductance. 

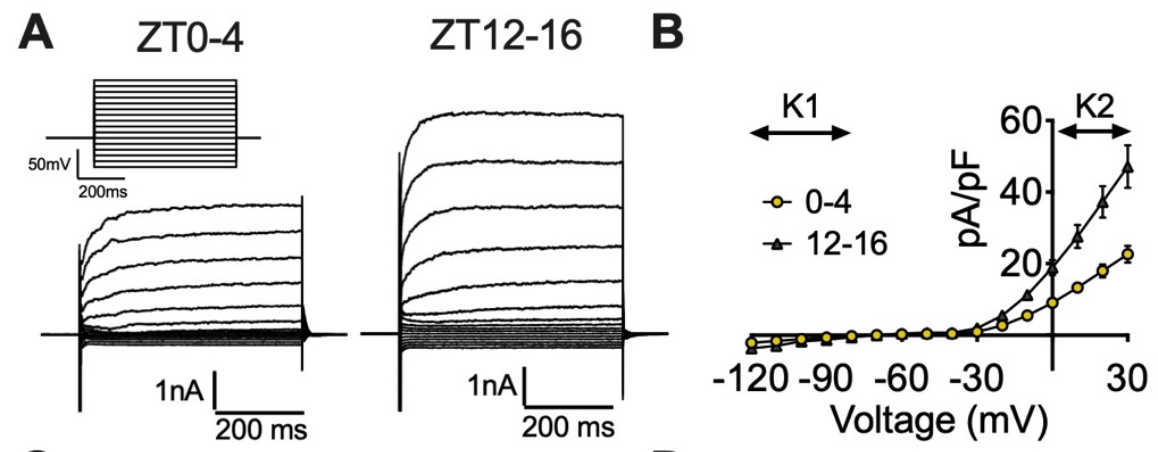

C

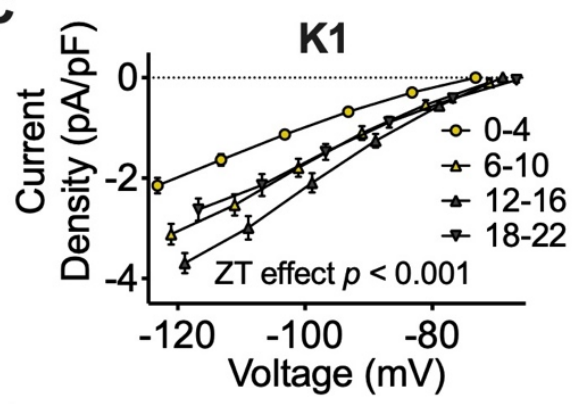

E

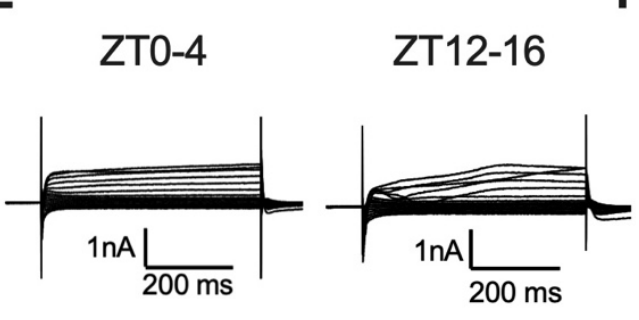

D

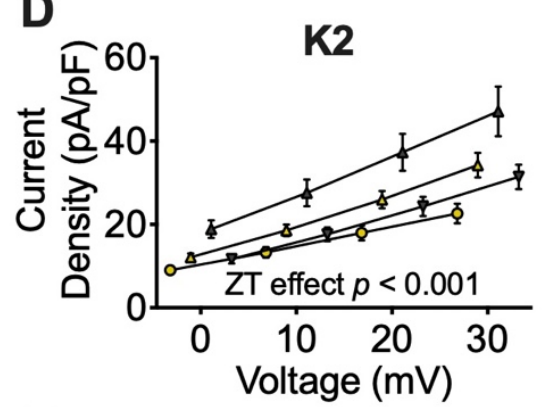

F

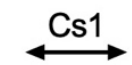

G

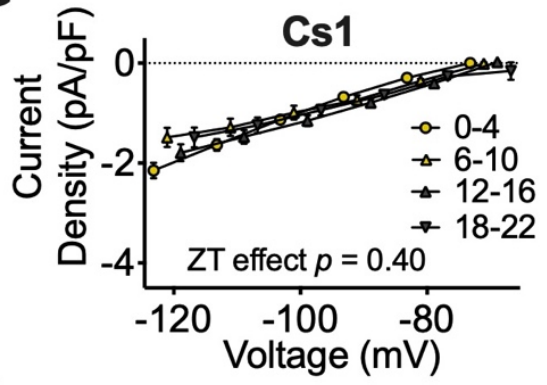

I

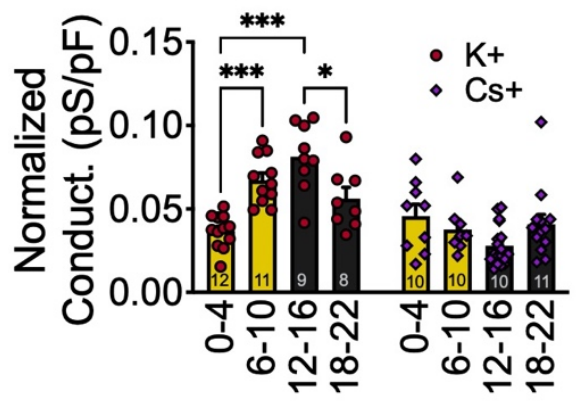

$\mathrm{H}$
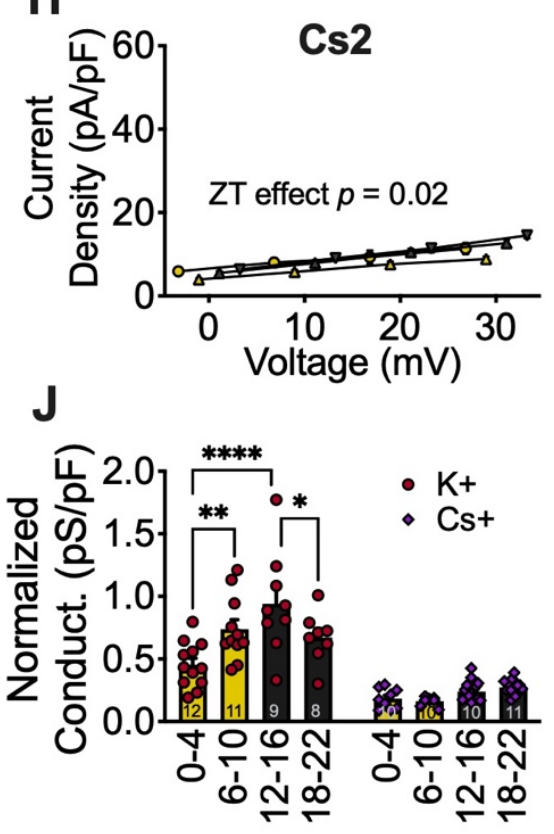
Figure 5. Membrane conductances are mediated by $\mathrm{K}+$ channels in males

(A) Voltage-step protocol (top left) and representative voltage-step traces of I-V relationship at ZT 04 (left) and 12-16 (right) in male mice. (B) Averaged I-V relationship for ZT0-4 and 12-16 (normalized to cell capacitance) with a $\mathrm{K}^{+}$internal solution. (C) Current density of $\mathrm{K} 1$ and (D) $\mathrm{K} 2 \mathrm{I}-\mathrm{V}$ relationships at ZT0-4, 6-10, 12-16, and 18-22. (E) Representative voltage-step traces of (F) I-V relationship at ZT 0-4 (left) and 12-16 (right) with a Cs+ internal solution. (G) Current density of Cs1 and (H) Cs2 I-V relationships at each ZT bin. (I) Comparison each ZT bin for K1 and Cs1, and (J) K2 and Cs2 normalized cell conductance. Two-way ANOVA for main effects and interaction, with a within group Tukey post-hoc analysis for ZT bin, voltage, and internal solution. Error bars represent $\pm 95 \% \mathrm{Cl}$ and $\mathrm{N}$-values for recorded cells are inset in bars. ${ }^{*} p<0.05,{ }^{* *} p<0.01,{ }^{* * *} p<0.001$. Exact $p$-values, mouse $\mathrm{N}$-values, and analysis in Figure 5 - source data 1.

\section{Time-of-day alters excitability of pIPFC pyramidal neurons}

To understand the functional implications of daily rhythms in RMP and postsynaptic ion channel function for information throughput, we tested how time-of-day impacts action potential dynamics. We utilized a 10 pA current injection protocol to evoke action potentials at ZT0-4, 6-10, 12-16, and 18-22 and observed a main effect of time for membrane potential threshold of action potential firing, with posthoc analysis revealing an increased threshold for firing late in the dark period (ZT18-22) when compared to ZT6-10 in male mice (Figure 6A-C). Consistent with the null effect of time on RMP in female mice, there was no effect of time on action potential threshold (Figure 6C). Further, although there was no effect on amount of current needed to elicit an action potential (rheobase), once rheobase was reached, subsequent current injections evoked action potential firing at a lower frequency during the dark period (Figure 6D, E). Although we did not observe a time-of-day effect on action potential amplitude or half-width in male mice (Figure 6F-G), decay tau was reduced at ZT18-22, a component of action potential firing that is modulated to a large extent by $\mathrm{K}^{+}$channels (Figure 6E). These data suggest that pIPFC pyramidal neurons are not only more hyperpolarized during the light period, but are 
bioRxiv preprint doi: https://doi.org/10.1101/2022.0127.478010; this version posted January 28, 2022. The copyright holder for this preprint (which was not certified by peer review) is the author/funder, who has granted bioRxiv a license to display the preprint in perpetuity. It is made available under aCC-BY-NC-ND 4.0 International license.

226 functionally more difficult activate, requiring larger depolarizations to elicit action potentials and relay

227 information downstream.
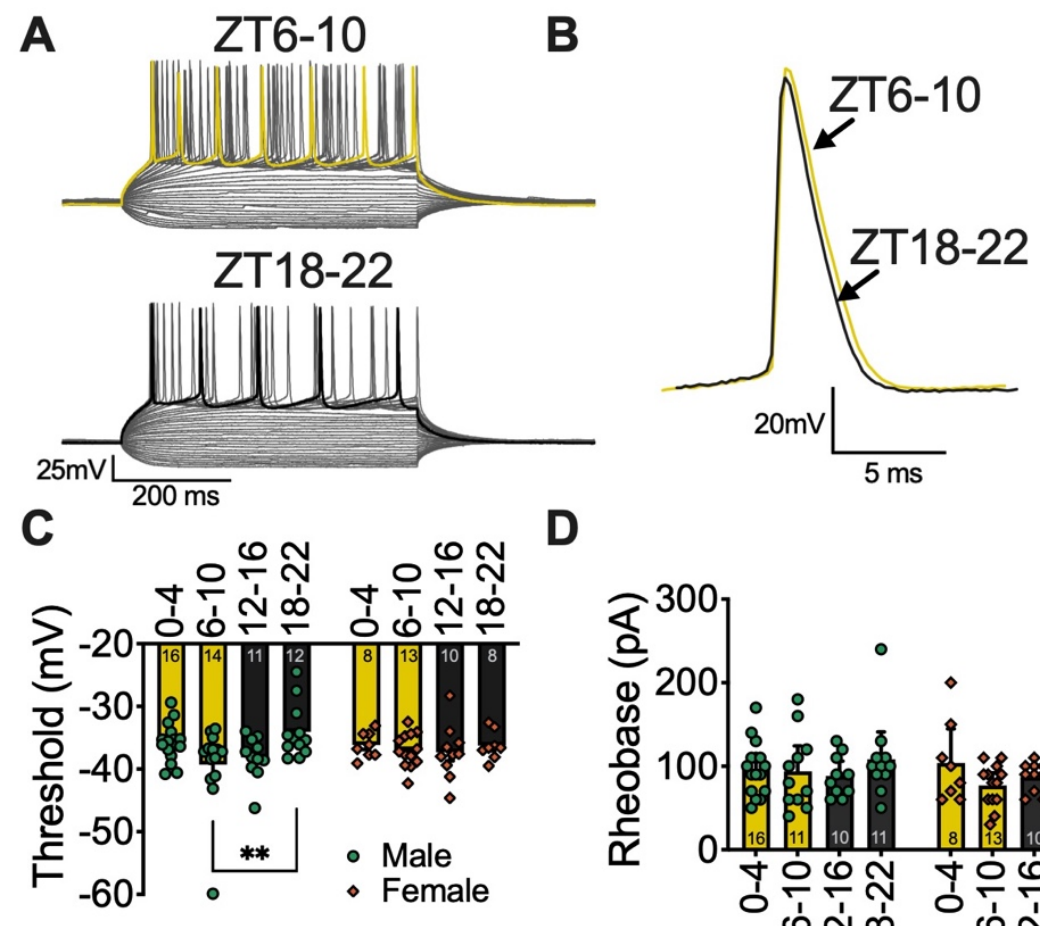

E
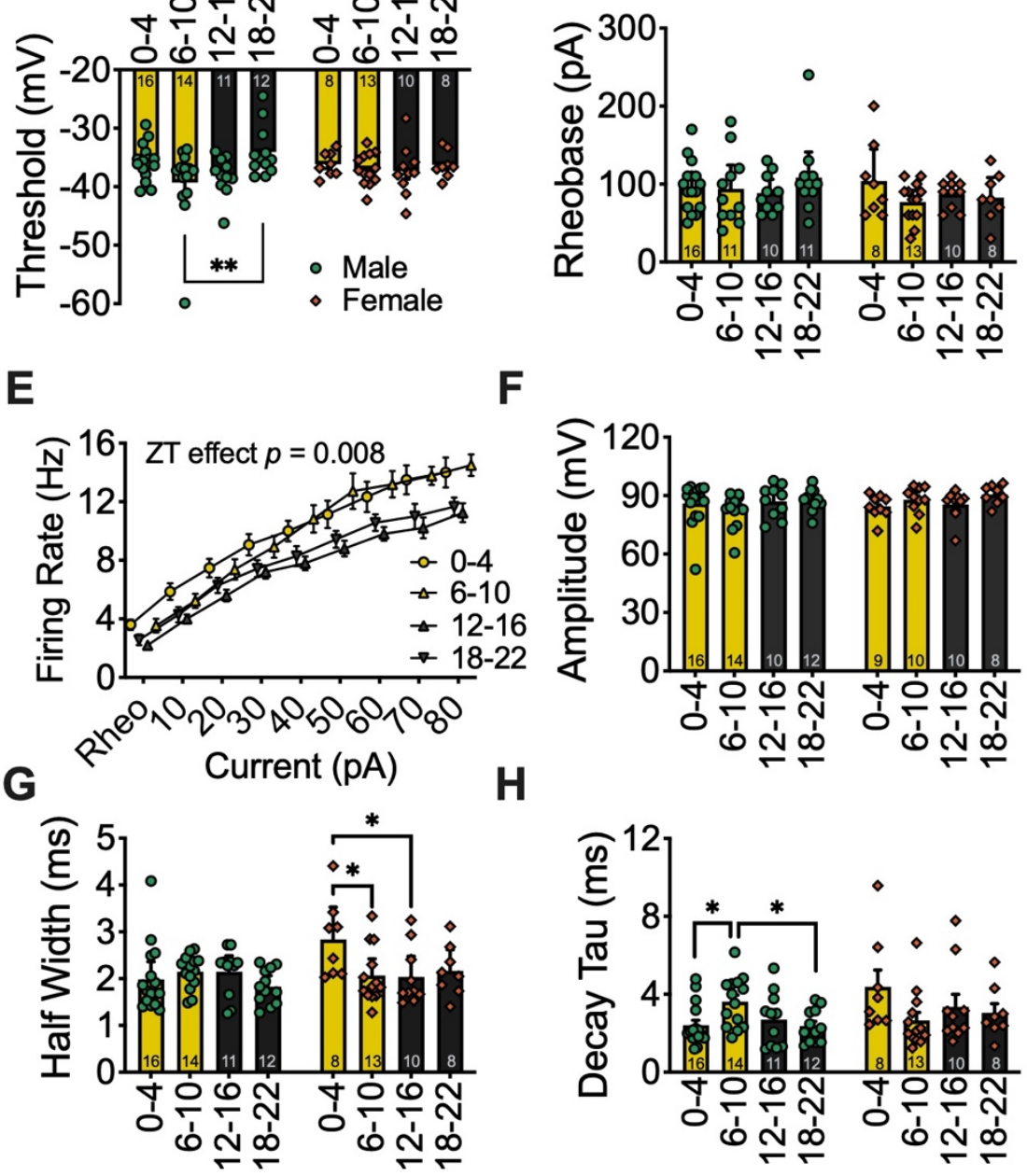
Figure 6. Time-of-day differences in action potential dynamics. (A) Representative trace of current step recording with maximal AP firing highlighted at ZT6-10 (top; yellow) and 18-22 (bottom; black) and (B) individual evoked APs in male mice. (C) Mean AP threshold (D) Rheobase, (E) evoked firing rate (from rheobase; male mice), (F) amplitude, (G) half width, and $(\mathbf{H})$ decay tau at each ZT bin in male and female mice. Error bars represent $\pm 95 \% \mathrm{Cl}$. Two-way ANOVA for main effects and interaction, with a within group Tukey post-hoc analysis for ZT bin and/or current injection. * $p<0.05$, ${ }^{* *} p<0.01$. Number of cells included in for each bar. Exact $p$-values, mouse $\mathrm{N}$-values, and analysis in Figure 6 - source data 1. 


\section{Discussion}

The suprachiasmatic nucleus $(\mathrm{SCN})$ is the central circadian clock in mammals and regulates daily changes in gene expression and activity throughout the brain (M. H. Hastings, Reddy, and Maywood 2003). However, it has become clear that numerous brain regions also display endogenous daily rhythms. A major hypothesis in the field is that these extra-SCN clocks and the SCN operate synergistically to drive daily rhythms in nearly all components of physiology and behavior, which is why their disruption has numerous physiological and psychological consequences, including exacerbated metabolic and mood disorders (Otsuka et al. 2020; McCarthy and Welsh 2012; Morris et al. 2015; Bechtold, Gibbs, and Loudon 2010; Karatsoreos 2012). While there have been several well executed studies that demonstrate the importance of circadian rhythms on neurophysiological function in the hippocampus and brainstem, none have included the PFC, and all have largely focused on extracellular field recordings (Chaudhury, Wang, and Colwell 2005; Loh et al. 2015; Paul et al. 2020; Chrobok et al. 2021; McMartin et al. 2021). Thus, previous studies have not shed light on the fundamental electrophysiological processes at the cellular level that are affected by time-of-day. In this study we present four main findings. First, layer 2/3 pIPFC pyramidal neurons in male mice are hyperpolarized during the early portion of the dark period when compared to the latter portion of the light period. Second, time-of-day impacts excitatory and inhibitory inputs onto pIPFC pyramidal neurons, with clear sex differences in excitatory inputs. Third, we demonstrate that male mice display distinct changes in ion channel activity and action potential kinetics, with male mice having an increased action potential firing threshold and decreased decay tau during portions of the dark period. Fourth, we identify that changes in $\mathrm{K}^{+}$channel activity serves as a potential mechanism underlying time-of-day changes in the RMP and action potential firing rates of pIPFC pyramidal neurons. By identifying the intrinsic properties and synaptic inputs of pIPFC pyramidal neurons, these findings allow us to better understand the relationship between circadian rhythms, PFC circuitry and its associated behaviors.

Changes in PFC function underly numerous psychiatric disorders including bipolar, posttraumatic stress disorder (PTSD), attention deficit disorder, and deficits in learning and memory (Popoli 
et al. 2012; Sotres-Bayon, Cain, and LeDoux 2006; Miller and Cohen 2001; Xu et al. 2019). There is growing evidence of links between circadian rhythms and PFC function (Otsuka et al. 2020; Woodruff et al. 2018; Hou et al. 2022; Harkness et al. 2021). Previous work from our group has demonstrated that extracellular lactate (a functional output of neural metabolism) shows circadian rhythms in the medial (m) PFC, and that environmental circadian disruption alters the morphology of medial mPFC neurons and affects PFC mediated behaviors (Wallace et al. 2020; Karatsoreos et al. 2011). However, the

263 studies presented here are the first to explore whether cell autonomous activity and synaptic inputs 264 onto PFC neurons are rhythmic.

Our finding that the resting state of pIPFC neurons is more hyperpolarized during the dark period, when the animals are awake and active, suggests that these neurons are less active and require a higher degree of information input before eliciting a response and sending downstream signals to other brain regions. On the surface, it seems counter-intuitive that pIPFC pyramidal neurons would be more inactive during the dark period, when these animals are active and engaging with their environment, than the light (inactive) period. A functional hypothesis for this finding is that stronger gating during the wake period serves as a necessary mechanism for selective informational throughput in response to environmental stimuli. Information filtering is paramount to having a proper behavioral output, and too low of a threshold may result in overactivation as the animal engages with its environment. For example, pharmacological studies have demonstrated that activation of the pIPFC with neurotensin agonists or the sodium channel activator veratrine lead to anxiogenic behaviors, likely through increased glutamate release (Li, Chang, and Xi 2021; Petrie et al. 2004; Saitoh et al. 2014). Notably, 278 in line with other studies demonstrating that in layer 2/3 cortical neurons, excitatory inputs are 279 increased during spontaneous wakefulness and sleep deprivation occurring during the light (inactive) 280 period (Liu et al. 2010). While it seems contradictory that these neurons simultaneously receive more 281 excitatory inputs and become more hyperpolarized, it is aligned with the proposal that these neurons 282 require stronger gating mechanisms during the active period, as more information is being sent to these 
283 neurons and it is critical that these incoming stimuli are somewhat filtered so only the strongest signals

284 are relayed further downstream.

285 Neurophysiological sex differences in the PFC, and their respective behavioral outputs, are well 286 documented and partly attributed to differences in synaptic signaling (Andrade et al. 2012; de Velasco

287 et al. 2015). While exploring the effects of time-of-day on these fundamental properties of PFC cells, we

288 fully embraced inclusion of both males and females, given the significant work demonstrating that

289 inclusion of both sexes (particularly inclusion of females) can reveal important new concepts and

290 understanding about brain function (Shansky and Murphy 2021). While not designed explicitly as a sex-

291 differences study, our results demonstrate that female mice had less excitatory inputs than males, yet

292 these inputs resulted in much larger postsynaptic currents, likely due to sex differences in glutamate

293 receptor expression and basal release (Perry et al. 2021). There are also sex differences in response to

294 environmental and pharmacological stressors, which are due in part to circulating sex hormones (Yuen,

295 Wei, and Yan 2016). For example, when compared to male rats, females in proestrus display a lower

296 threshold for impaired working memory after PFC injections of benzodiazepine inverse agonists that

297 activate the stress system, but this effect does not persist during estrus, when circulating estrogen

298 levels are lower (Shansky et al. 2004). Further, gonadal hormones underly sex differences in mPFC

299 dendritic growth, microglia activity, and astrocyte morphology in response to stress (Bollinger et al.

300 2019). It should be noted that there is a report that basal PFC glutamate release is higher in females,

301 which seems opposite to our findings, but is likely due to experimental differences, as these studies

302 differ in species, PFC layers, electrophysiological solutions, and time-of-day (Pena-Bravo et al. 2019).

303 We speculate that if the underlying mechanisms that mediate information throughput and plasticity are

304 fundamentally different in males and females, and the basal tone of excitatory inputs is relatively low in

305 females, then time-of-day changes in information filtering may not be as crucial to optimal pIPFC

306 function in female mice.

307 GABAergic interneurons are highly involved in PFC function and relay information between

308 multiple regions of the PFC (Saffari et al. 2016; Hu, Gan, and Jonas 2014; Anderson et al. 2021). In the

309 hippocampus, GABAergic inputs onto CA1 pyramidal neurons regulate action potential firing frequency 
310 in response to current injections, with a higher inhibitory tone during the light cycle (Fusilier et al. 2021;

311 Albers et al. 2017). Significantly, we found that in the pIPFC there was no effect of sex or time-of-day

312 on spontaneous inhibitory inputs. This suggests that presynaptic inhibitory and excitatory inputs are not

313 the primary regulator of resting state or information throughput in layer 2/3 pIPFC pyramidal neurons.

314 Instead, this supports the notion that basal inhibitory tone remains relatively constant throughout the

$31524 \mathrm{~h}$ day and, in male mice, a postsynaptic cell endogenous mechanism is responsible for maintaining

316 proper information filtering when these neurons are challenged by the large increase of excitatory

317 signals that come in during the active period.

318 Although the frequency and amplitude of spontaneous inhibitory inputs did not change when

319 probed by individual ZT bins, further investigation revealed that when grouped by the light/dark cycle,

320 the strength (amplitude) of inhibitory inputs was stronger during the dark (active) period, specifically in

321 male mice. GABA receptors interact with postsynaptic ion channels and there are sex differences in the

322 expression of GABA receptor subunits, as well as how they interact with ion channels. For example, the

323 steroid hormone progesterone increases the expression of the GABA receptor subunit $\alpha 1$ in the PFC

324 of rodents, and in humans, alcoholism is suggested to result in larger decreases of cortical GABA(A)

325 receptor subunits in females than males (Andrade et al. 2012; Janeczek et al. 2020). After investigating

326 GABAergic signaling localized at the synapse and isolated from upstream activity, we confirmed that

327 inhibitory postsynaptic currents are stronger early in the dark period in male mice. Interestingly, female

328 mice displayed a large increase in inhibitory inputs and strength late in the light period, a finding that

329 requires future studies to fully understand its functional implications.

330 In total, our findings point toward a postsynaptic mechanism underlying daily changes in the

331 physiology of layer 2/3 pIPFC pyramidal neurons and previous work has shown that sleep deprivation

332 can alter the intrinsic excitability of layer 5 PFC pyramidal neurons (Yan et al. 2011). This prompted us

333 to explore how time-of-day impacts intrinsic postsynaptic properties such as ionic currents and overall

334 conductance. At hyperpolarized voltage steps below the reversal potential of $\mathrm{K}^{+}$, time-of-day did have a

335 modest impact the conductance and current density of pIPFC pyramidal neurons in female mice

336 between the transition from the dark to light period. However, at depolarizing voltages greater than the 
337 mean RMP and action potential threshold there was no time-of-day effect. This further supports the 338 notion that while physiological daily rhythms do exist in pIPFC pyramidal neurons of female mice, they

339 have little impact on overall resting state and information throughput in response to electrical activity

340 and synaptic inputs. In contrast, there was a large effect on the current density and conductance of

341 pyramidal neurons in male mice. Specifically, when these neurons were hyperpolarized below the

342 equilibrium potential for $\mathrm{K}^{+}$, we discovered that current density increased throughout the light period,

343 peaking between the late period and early dark period. This effect translated into an overall increase in

344 conductance, and given that conductance was highest around the beginning of the active period, when

345 these neurons are most hyperpolarized, we presumed that this was due to an increased number of

346 open $\mathrm{K}^{+}$channels and the outflow of $\mathrm{K}^{+}$cations (outward current). Consistent with this prediction, when

347 we replaced $\mathrm{K}^{+}$with $\mathrm{Cs}^{+}$in our internal recording solution (to block $\mathrm{K}^{+}$channel mediated outward

348 currents), the time-of-day effect on current density and conductance was completely abolished at

349 voltages near or below the $\mathrm{K}^{+}$equilibrium potential. Although internal $\mathrm{Cs}^{+}$was not sufficient to block the

350 time-of-day effect on current density at depolarized voltage greater than the $\mathrm{K}^{+}$equilibrium potential, it

351 greatly reduced overall current density and conductance. Moreover, previous studies demonstrate that

352 internal $\mathrm{Cs}^{+}$is not sufficient to block the inward $\mathrm{K}^{+}$currents expected at voltages above the $\mathrm{K}^{+}$

353 equilibrium potential (Adelman and Senft 1966).

354 To understand the functional relevance of these postsynaptic changes in ion channel function

355 and resting state, it was necessary to determine how time-of-day impacts action potential dynamics as

356 action potential firing is a functional measure for information throughput. Notably, this measure changes

357 with time-of-day in the hippocampus, and in response to sleep deprivation in the PFC (Fusilier et al.

358 2021; Yan et al. 2011). Action potentials are dependent on voltage-gated ion channels, and changes in

$359 \mathrm{~K}^{+}$channel activity can alter action potential firing threshold and kinetics. Consistent with our

360 interpretation that, in male mice, there is a stronger gating mechanism to filter incoming signals during

361 the active period, we discovered that the threshold for action potential firing was increased during the

362 active period. These data suggest that layer 2/3 pIPFC pyramidal neurons are not only more

363 hyperpolarized during the light period, but are functionally more difficult to activate, requiring much 
364 larger depolarizations to elicit action potentials and relay information downstream. Though somewhat 365 speculative, this could affect a wide range of behaviors, including emotionality, a notion supported by 366 work demonstrating that pharmacological activation of pIPFC neurons induces anxiogenic activity in 367 mice, and acute stress enhances glutamatergic transmission in the PFC (Yuen et al. 2009; Li, Chang, 368 and Xi 2021; Saitoh et al. 2014). Further, alcohol is a common drug of abuse in those suffering from 369 PFC associated affective pathologies, and in vivo electrophysiology studies show that alcohol preferring 370 rats have higher baseline neural firing in the PFC (Linsenbardt and Lapish 2015). Combined, we believe this work demonstrates the importance of understanding how daily 372 rhythms impact neural function, which is necessary to fully grasp the relationships between brain and 373 behavior. It is critical to recognize that the mPFC is heterogeneous at the anatomical and physiological 374 levels, with consequences for behavior (Moorman et al. 2015). Our work suggests that even when 375 looking at the fundamental properties of cellular function in the mPFC, perhaps we need to add 376 heterogeneity at the temporal level as well. To fully appreciate the relationship between brain, behavior, 377 and daily rhythms, future studies are required to determine how these rhythms impact communication 378 with extra- (such as the hippocampus and amygdala) and intra- (such as the infralimbic) PFC regions. 379 Additionally, future studies are necessary to determine exactly which ion channels are mediating daily 380 changes in PFC function and how environmental factors that alter whole-animal physiology and 381 behavior, such as circadian disruption, may impact these circuits. Given the impact of time-of-day on 382 neuronal function in the PFC, the work presented here also has significant implications for incorporating 383 time-of-day into the application of pharmacological and behavioral interventions for mental health 384 disorders, and opens the door to similar questions in brain regions outside the PFC. 


\section{Methods}

387 Animals

Amherst Institutional Care and Use Committee in accordance with the U.S. Public Health Service Policy

on Humane Care and Use of Laboratory Animals and the National Institutes of Health Guide for the

391 Care and Use of Laboratory Animals. Male and female wild-type mice (Charles River, Wilmington, MA,

392 USA) on a C57BL/6J background were used for these studies. All mice were group-housed in light

393 boxes at $25^{\circ} \mathrm{C}$, under a 12/12-hr light/dark (LD) cycle, with food and water available ad libitum. Light

394 box LD cycles were offset so that experiments from each ZT bin occurred at the same time each day.

395 Mice ages 10-16 weeks were used for these studies. For electrophysiology studies mice were anesthetized in a chamber with isoflurane before euthanasia by decapitation.

Brain slice electrophysiology

Two mice were simultaneously euthanized 1-hr prior to their ZT bin (i.e., mice were euthanized at ZT23 for recording bin ZT0-4). After euthanasia, brains were immediately removed and the forebrain was blocked while bathing in a $0-4^{\circ} \mathrm{C}$ oxygenated N-methyl-D-glucamine (NMDG) - 4-(2-hydroxyethyl)1-piperazineethanesulfonic acid (HEPES) cutting solution composed of (mM): $92 \mathrm{NMDG}, 2.5 \mathrm{KCl}, 1.25$

$403 \mathrm{NaH}_{2} \mathrm{PO}_{4}, 30 \mathrm{NaHCO}_{3}, 3$ sodium pyruvate, 2 thiourea, $20 \mathrm{HEPES}, 10 \mathrm{MgSO}_{4}, 0.5 \mathrm{CaCl}_{2}, 25$ glucose, 40420 sucrose. Cutting solution was brought to $\mathrm{pH} 7.4$ with $\sim 17 \mathrm{~mL}$ of $5 \mathrm{M} \mathrm{HCl}$ (Ting et al. 2018). The 405 forebrains were mounted adjacent to each other and sectioned simultaneously on a vibratome 406 (VT1200S, Leica Biosciences, Buffalo Grove, IL, USA) with a sapphire knife (Delaware Diamond 407 Knives, Wilmington, DE, USA) yielding roughly three slices containing the PFC from each (250- $\mu \mathrm{m})$ per 408 mouse. Slices were transferred and allowed to recover for 30-45 min in room temperature recording 409 artificial cerebrospinal fluid (aCSF) solution composed of (mM): $124 \mathrm{NaCl}, 3.7 \mathrm{KCl}, 2.6 \mathrm{NaH}_{2} \mathrm{PO}_{4}, 26$

$410 \mathrm{NaHCO}_{3}, 2 \mathrm{CaCl}_{2}, 2 \mathrm{MgSO}_{4}, 10$ glucose. aCSF had a final pH of 7.3-7.4, osmolarity of 307-310

411 mOsmos, and was continuously bubbled using $95 \% \mathrm{O}_{2} / 5 \% \mathrm{CO}_{2}$. For recordings, brain slices were 412 transferred to a perfusion chamber containing aCSF maintained at $34-37^{\circ} \mathrm{C}$ with a flow rate of $1 \mathrm{~mL} / \mathrm{min}$. 
413 Neurons were visualized using an upright microscope (Zeiss Axoskop 2, Oberkochen, Germany).

414 Recording electrodes were back-filled with experiment-specific internal solutions as follows (mM):

415 Current-clamp and spontaneous (s) excitatory postsynaptic currents (EPSCs); 125 K-gluconate, 10

$416 \mathrm{KCl}, 10 \mathrm{NaCl}, 5 \mathrm{HEPES}, 10 \mathrm{EGTA}, 1 \mathrm{MgCl}_{2}, 3 \mathrm{NaATP}$ and $0.25 \mathrm{NaGTP}$ (liquid-junction potential (LJP)

$417=\sim 14.5 \mathrm{mV}$ ). Voltage-clamp spontaneous inhibitory postsynaptic currents (sIPSCs); $140 \mathrm{CsCl}, 5 \mathrm{MgCl}_{2}$,

$4181 \mathrm{EGTA}, 10 \mathrm{HEPES}, 3 \mathrm{NaATP}$, and $0.25 \mathrm{NaGTP}$ (LJP = $4.2 \mathrm{mV}$ ). All internal solutions were brought to

$419 \mathrm{pH} 7.3$ using $\mathrm{KOH}$ (current-clamp and EPSC) or CsOH (IPSC) at 301-304 mOsm. EPSCs were

420 recorded in the presence of the GABA receptor antagonist bicuculline $(30 \mu \mathrm{M})$. sIPSCs were recorded

421 in the presence the competitive a-amino-3-hydroxy-5-methyl-4-isoxazolepropionic acid (AMPA)/kainate

422 receptor antagonist 6-cyano-7-nitroquinoxaline-2,3-dione (CNQX; $10 \mu \mathrm{M})$, the selective N-Methyl-d-

423 aspartate (NMDA) receptor antagonist (2R)-amino-5-phosphonovaleric acid (APV; $50 \mu \mathrm{M})$, and the

424 glycine antagonist strychnine $(2 \mu \mathrm{M})$. Miniature $(\mathrm{m})$ IPSCs had the addition of the voltage-gated sodium

425 channel (VGSC) blocker tetrodotoxin (TTX; $1 \mu \mathrm{M})$. Patch electrodes with a resistance of 3-5M $\Omega$ were

426 guided to neurons with an MPC-200-ROE controller and MP285 mechanical manipulator (Sutter

427 Instruments, Novato, CA, USA). Patch-clamp recordings were collected through a UPC-10 USB dual

428 digital amplifier and Patchmaster NEXT recording software (HEKA Elektronik GmbH, Reutlingen,

429 Germany). All voltage-clamp recordings were obtained at $\mathrm{V}_{\mathrm{H}}=-70 \mathrm{mV}$. Current clamp voltage-step

430 protocols were performed from the cell endogenous resting membrane potential, and used 500ms 10pA

431 steps from $-100 p A$ to $+190 p A$. Voltage clamp current-step protocols were performed from $V_{H}=-70 m V$,

432 and used $10 \mathrm{mV}$ steps from $-120 \mathrm{mV}$ to $+30 \mathrm{mV}$. All compounds were obtained from Tocris Cookson,

433 Cayman Chemical, and Sigma Aldrich.

$434 \quad$ Individual recording locations were plotted (with neurons outside of the target area excluded

435 from analysis) as well as to qualitatively confirm an equal distribution of recording sites between

436 Zeitgeber (ZT) bins 0-4, 6-10, 12-16, and 18-22 (Figure 1 - figure supplement 1A-D). A small

437 percentage ( $20 \%)$ of all recorded neurons had unique characteristics in resting membrane properties,

438 spontaneous excitatory postsynaptic currents (sEPSCs), and action potential dynamics that were

439 independent of time-of-day (hereafter Type II neurons; Figure 1 - figure supplement 2A-I). Most 
440 notably, compared to Type I (most abundant) neurons, Type II neurons (less abundant) displayed a

441 much higher action potential velocity and hyperpolarizing decay current (Figure 1 - figure supplement

442 2A, B). They also had a more depolarized resting membrane potential (RMP) and decreased action

443 potential firing threshold (Figure 1 - figure supplement 2E, F). Due to these clear qualitative and

444 quantitative differences independent of ZT bin, and that they represented a small proportion of recorded

445 neurons, we excluded the far less abundant Type II neurons from analysis in our following experiments.

Statistical Analysis

For sEPSCs, only neurons with holding currents not exceeding $100 \mathrm{pA}$ at $\mathrm{V}_{\mathrm{H}}=-70 \mathrm{mV}$ for the 10min control period (input resistance $>70 \mathrm{M} \Omega$ ) were studied further. Neurons were not considered for

451 further analysis if series resistance exceeded $50 \mathrm{M} \Omega$ or drifted $>10 \%$ during baseline. Rheobase was 452 calculated as the first current step to elicit an action potential and action potential dynamics (threshold, 453 decay tau, and half-width) were obtained from the first evoked action potential to avoid variance in ion 454 channel function due to repeated action potential firing. G*Power 3.0 software (Franz Faul, Uni Kiel, 455 Germany) was used to conduct our power analysis, for a $p$ value of $<0.05$ with $90 \%$ power. Adequate 456 sample sizes were based upon expected effect sizes from similar experiments. Raw data files were 457 analyzed in the Patchmaster NEXT software or converted using ABF Utility (Synaptosoft) for analysis in 458 Clampfit (Molecular Devices, San Jose, CA, USA). N-values for analysis and presented in figures 459 represent individual cells. To control for biological variability between groups $\mathrm{N}=4-8$ mice per group 460 (see figure source data). Statistical comparison of effects between each time-period was made using a 461 full model two-way ANOVA (column, row, and interaction effects) for comparison of the current-voltage 462 relationships and comparing the interaction and main effect of time and sex or internal solution. For all 463 experiments, error bars are presented as mean $\pm 95 \%$ confidence interval $(\mathrm{Cl})$. Statistics were 464 calculated using Prism 9 (Graphpad Software, San Diego, CA, USA). 
bioRxiv preprint doi: https://doi.org/10.1101/2022.0127.478010; this version posted January 28, 2022. The copyright holder for this preprint

(which was not certified by peer review) is the author/funder, who has granted bioRxiv a license to display the preprint in perpetuity. It is made available under aCC-BY-NC-ND 4.0 International license.

\section{Acknowledgements}

466 We would like to acknowledge and thank Dr. James Peters at Washington State University for his

467 invaluable input and discussions during the preparation of this manuscript.

468

469 Grants

470 This work was supported by a CAREER grant 1553067 by the National Science Foundation, and R01

471 DK119811 from the National Institutes of Health to INK.

472

473 Competing Interests

474 BLR, JW and INK declare no competing financial interests.

475 


\section{References}

Abe, Michikazu, Erik D. Herzog, Shin Yamazaki, Marty Straume, Hajime Tei, Yoshiyuki Sakaki, Michael Menaker, and Gene D. Block. 2002. "Circadian Rhythms in Isolated Brain Regions." The Journal of Neuroscience 22 (1): 350-56. https://doi.org/10.1523/JNEUROSCI.22-0100350.2002 .

Adelman, W. J., and J. P. Senft. 1966. "Voltage Clamp Studies on the Effect of Internal Cesium Ion on Sodium and Potassium Currents in the Squid Giant Axon." The Journal of General Physiology 50 (2): 279-93. https://doi.org/10.1085/jgp.50.2.279.

Aerde, Karlijn I. van, and Dirk Feldmeyer. 2015. "Morphological and Physiological Characterization of Pyramidal Neuron Subtypes in Rat Medial Prefrontal Cortex." Cerebral Cortex 25 (3): 788-805. https://doi.org/10.1093/cercor/bht278.

Albers, Elliott H., James C. Walton, Karen L. Gamble, John K. McNeill, and Daniel L. Hummer. 2017. "The Dynamics of GABA Signaling: Revelations from the Circadian Pacemaker in the Suprachiasmatic Nucleus." Frontiers in Neuroendocrinology 44 (January): 35-82. https://doi.org/10.1016/j.yfrne.2016.11.003.

Albrecht, Anne, and Oliver Stork. 2017. "Circadian Rhythms in Fear Conditioning: An Overview of Behavioral, Brain System, and Molecular Interactions." Neural Plasticity 2017: 3750307. https://doi.org/10.1155/2017/3750307.

Anderson, Eden M., Skyler Demis, Hunter D’Acquisto, Annabel Engelhardt, and Matthew Hearing. 2021. "The Role of Parvalbumin Interneuron GIRK Signaling in the Regulation of Affect and Cognition in Male and Female Mice." Frontiers in Behavioral Neuroscience 15 (March): 621751. https://doi.org/10.3389/fnbeh.2021.621751.

Andrade, Susie, Bruno D. Arbo, Bruna A. M. Batista, Alice M. Neves, Gisele Branchini, IIma S. Brum, Helena M. T. Barros, Rosane Gomez, and Maria Flavia M. Ribeiro. 2012. "Effect of Progesterone on the Expression of GABA(A) Receptor Subunits in the Prefrontal Cortex of Rats: Implications of Sex Differences and Brain Hemisphere." Cell Biochemistry and Function 30 (8): 696-700. https://doi.org/10.1002/cbf.2854. 
503 Bano-Otalora, Beatriz, Matthew J. Moye, Timothy Brown, Robert J. Lucas, Casey O. Diekman, and Mino Dc Belle. 2021. "Daily Electrical Activity in the Master Circadian Clock of a Diurnal Mammal." ELife 10 (November): e68179. https://doi.org/10.7554/eLife.68179.

Bechtold, D. A., J. E. Gibbs, and A. S. Loudon. 2010. "Circadian Dysfunction in Disease." Trends Pharmacol Sci 31 (5): 191-98. https://doi.org/S0165-6147(10)00003-9 [pii] 10.1016/j.tips.2010.01.002.

Bollinger, Justin L., Isabella Salinas, Emily Fender, Dale R. Sengelaub, and Cara L. Wellman. 2019. "Gonadal Hormones Differentially Regulate Sex-Specific Stress Effects on Glia in the Medial Prefrontal Cortex." Journal of Neuroendocrinology 31 (8): e12762. https://doi.org/10.1111/jne.12762.

Chaudhury, D., L. M. Wang, and C. S. Colwell. 2005. "Circadian Regulation of Hippocampal Long-Term Potentiation." J Biol Rhythms 20 (3): 225-36. https://doi.org/20/3/225 [pii] $10.1177 / 0748730405276352$.

Chrobok, Lukasz, Jasmin D. Klich, Jagoda S. Jeczmien-Lazur, Kamil Pradel, Katarzyna PalusChramiec, Anna M. Sanetra, Hugh D. Piggins, and Marian H. Lewandowski. 2021. "Daily Changes in Neuronal Activities of the Dorsal Motor Nucleus of the Vagus under Standard and High-Fat Diet." The Journal of Physiology, May. https://doi.org/10.1113/JP281596.

Chun, Lauren E., Elizabeth R. Woodruff, Sarah Morton, Laura R. Hinds, and Robert L. Spencer. 2015. "Variations in Phase and Amplitude of Rhythmic Clock Gene Expression across Prefrontal Cortex, Hippocampus, Amygdala, and Hypothalamic Paraventricular and Suprachiasmatic Nuclei of Male and Female Rats." Journal of Biological Rhythms 30 (5): 417-36. https://doi.org/10.1177/0748730415598608.

Deng, Wei-Ke, Xing Wang, Hou-Cheng Zhou, and Fei Luo. 2019. "L-Type Ca2+ Channels and Charybdotoxin-Sensitive Ca2+-Activated K+ Channels Are Required for Reduction of GABAergic Activity Induced by B2-Adrenoceptor in the Prefrontal Cortex." Molecular and Cellular Neurosciences 101 (December): 103410. https://doi.org/10.1016/j.mcn.2019.103410. 
529 Ferguson, Brielle R., and Wen-Jun Gao. 2018. "PV Interneurons: Critical Regulators of E/l Balance for Prefrontal Cortex-Dependent Behavior and Psychiatric Disorders." Frontiers in Neural Circuits

532 Fusilier, Allison R., Jennifer A. Davis, Jodi R. Paul, Stefani D. Yates, Laura J. McMeekin, Lacy K.

Harkness, John H., Angela E. Gonzalez, Priyanka N. Bushana, Emily T. Jorgensen, Deborah M. Hegarty, Ariel A. Di Nardo, Alain Prochiantz, et al. 2021. "Diurnal Changes in Perineuronal Nets and Parvalbumin Neurons in the Rat Medial Prefrontal Cortex." Brain Structure \& Function 226 Goode, Mugdha V. Mokashi, et al. 2021. "Dysregulated Clock Gene Expression and Abnormal Diurnal Regulation of Hippocampal Inhibitory Transmission and Spatial Memory in Amyloid Precursor Protein Transgenic Mice." Neurobiology of Disease 158 (October): 105454. https://doi.org/10.1016/j.nbd.2021.105454.

(4): 1135-53. https://doi.org/10.1007/s00429-021-02229-4.

Hastings, J. W. 2007. "The Gonyaulax Clock at 50: Translational Control of Circadian Expression." Cold Spring Harbor Symposia on Quantitative Biology 72 (January): 141-44.

https://doi.org/10.1101/sqb.2007.72.026.

Hastings, Michael H., Akhilesh B. Reddy, and Elizabeth S. Maywood. 2003. "A Clockwork Web: Circadian Timing in Brain and Periphery, in Health and Disease." Nature Reviews Neuroscience 4 (8): 649-61. https://doi.org/10.1038/nrn1177.

Helm, Barbara, Rachel Ben-Shlomo, Michael J. Sheriff, Roelof A. Hut, Russell Foster, Brian M. Barnes, and Davide Dominoni. 2013. “Annual Rhythms That Underlie Phenology: Biological TimeKeeping Meets Environmental Change." Proceedings of the Royal Society B: Biological Sciences 280 (1765): 20130016. https://doi.org/10.1098/rspb.2013.0016.

Hou, YuanYuan, YunLei Wang, ShaoFei Song, Yao Zuo, HaoJie Zhang, Chen Bai, HaiTao Zhao, and Tong Zhang. 2022. “Long-Term Variable Photoperiod Exposure Impairs the MPFC and Induces Anxiety and Depression-like Behavior in Male Wistar Rats." Experimental Neurology 347 (January): 113908. https://doi.org/10.1016/j.expneurol.2021.113908. 
555 Hu, H., J. Gan, and P. Jonas. 2014. “Fast-Spiking, Parvalbumin+ GABAergic Interneurons: From

Janeczek, Paulina, Natalie Colson, Peter R. Dodd, and Joanne M. Lewohl. 2020. "Sex Differences in

Jagannath, Aarti, Lewis Taylor, Zeinab Wakaf, Sridhar R. Vasudevan, and Russell G. Foster. 2017. "The Genetics of Circadian Rhythms, Sleep and Health." Human Molecular Genetics 26 (R2): R128-38. https://doi.org/10.1093/hmg/ddx240. the Expression of the A5 Subunit of the GABAA Receptor in Alcoholics with and without

Cirrhosis of the Liver." Alcoholism: Clinical and Experimental Research 44 (2): 423-34.

https://doi.org/10.1111/acer.14266.

Kalmbach, Brian E., and Darrin H. Brager. 2020. "Fragile X Mental Retardation Protein Modulates Somatic D-Type K+ Channels and Action Potential Threshold in the Mouse Prefrontal Cortex." Journal of Neurophysiology 124 (6): 1766-73. https://doi.org/10.1152/jn.00494.2020.

Karatsoreos, Ilia N. 2012. "Effects of Circadian Disruption on Mental and Physical Health." Curr Neurol Neurosci Rep 12 (2): 218-25. https://doi.org/10.1007/s11910-012-0252-0.

Karatsoreos, Ilia N., Sarah Bhagat, Erik B. Bloss, John H. Morrison, and Bruce S. McEwen. 2011. “Disruption of Circadian Clocks Has Ramifications for Metabolism, Brain, and Behavior.” Proceedings of the National Academy of Sciences 108 (4): 1657-62. https://doi.org/10.1073/pnas.1018375108.

Kawaguchi, Y., and Y. Kubota. 1997. "GABAergic Cell Subtypes and Their Synaptic Connections in Rat Frontal Cortex." Cerebral Cortex (New York, N.Y.: 1991) 7 (6): 476-86. https://doi.org/10.1093/cercor/7.6.476.

Körtner, Gerhard, and Fritz Geiser. 2000. “The Temporal Organization of Daily Torpor and Hibernation: Circadian and Circannual Rhythms." Chronobiology International 17 (2): 103-28. https://doi.org/10.1081/cbi-100101036.

Landgraf, Dominic, Jaimie E. Long, Christophe D. Proulx, Rita Barandas, Roberto Malinow, and David K. Welsh. 2016. "Genetic Disruption of Circadian Rhythms in the Suprachiasmatic Nucleus 

Psychiatry 80 (11): 827-35. https://doi.org/10.1016/j.biopsych.2016.03.1050.

Laroche, Serge, Therese M. Jay, and Anne-Marie Thierry. 1990. "Long-Term Potentiation in the Prefrontal Cortex Following Stimulation of the Hippocampal CA1/Subicular Region." Neuroscience Letters 114 (2): 184-90. https://doi.org/10.1016/0304-3940(90)90069-I.

Le Merre, Pierre, Sofie Ährlund-Richter, and Marie Carlén. 2021. "The Mouse Prefrontal Cortex: Unity in Diversity." Neuron 109 (12): 1925-44. https://doi.org/10.1016/j.neuron.2021.03.035.

Li, Bin, Lei-Lei Chang, and Kang Xi. 2021. "Neurotensin 1 Receptor in the Prelimbic Cortex Regulates Anxiety-like Behavior in Rats." Progress in Neuro-Psychopharmacology and Biological Psychiatry 104 (January): 110011. https://doi.org/10.1016/j.pnpbp.2020.110011.

Linsenbardt, David N., and Christopher C. Lapish. 2015. "Neural Firing in the Prefrontal Cortex During Alcohol Intake in Alcohol-Preferring 'P' Versus Wistar Rats." Alcoholism, Clinical and Experimental Research 39 (9): 1642-53. https://doi.org/10.1111/acer.12804.

Liu, Zhong-Wu, Ugo Faraguna, Chiara Cirelli, Giulio Tononi, and Xiao-Bing Gao. 2010. "Direct Evidence for Wake-Related Increases and Sleep-Related Decreases in Synaptic Strength in Rodent Cortex." Journal of Neuroscience 30 (25): 8671-75. https://doi.org/10.1523/JNEUROSCI.1409-10.2010.

Loh, Dawn H, Shekib A Jami, Richard E Flores, Danny Truong, Cristina A Ghiani, Thomas J O'Dell, and Christopher S Colwell. 2015. "Misaligned Feeding Impairs Memories." Edited by Joseph S Takahashi. ELife 4 (December): e09460. https://doi.org/10.7554/eLife.09460.

McCarthy, Michael J., and David K. Welsh. 2012. "Cellular Circadian Clocks in Mood Disorders:" Journal of Biological Rhythms, September. https://doi.org/10.1177/0748730412456367.

McMartin, Laura, Marianna Kiraly, H. Craig Heller, Daniel V. Madison, and Norman F. Ruby. 2021. "Disruption of Circadian Timing Increases Synaptic Inhibition and Reduces Cholinergic Responsiveness in the Dentate Gyrus." Hippocampus 31 (4): 422-34. https://doi.org/10.1002/hipo.23301. 
608

Miller, Earl K., and Jonathan D. Cohen. 2001. "An Integrative Theory of Prefrontal Cortex Function." Annual Review of Neuroscience 24 (1): 167-202. https://doi.org/10.1146/annurev.neuro.24.1.167.

Moorman, David E., Morgan H. James, Ellen M. McGlinchey, and Gary Aston-Jones. 2015. “Differential Roles of Medial Prefrontal Subregions in the Regulation of Drug Seeking." Brain Research, Role of corticostriatal circuits in addiction, 1628 (December): 130-46. https://doi.org/10.1016/j.brainres.2014.12.024.

Morris, C. J., J. N. Yang, J. I. Garcia, S. Myers, I. Bozzi, W. Wang, O. M. Buxton, S. A. Shea, and F. A. Scheer. 2015. "Endogenous Circadian System and Circadian Misalignment Impact Glucose Tolerance via Separate Mechanisms in Humans." Proc Natl Acad Sci U S A 112 (17): E222534. https://doi.org/10.1073/pnas.1418955112.

Otsuka, Tsuyoshi, Hue Thi Le, Akira Kohsaka, Fuyuki Sato, Hayato Ihara, Tomomi Nakao, and Masanobu Maeda. 2020. "Adverse Effects of Circadian Disorganization on Mood and Molecular Rhythms in the Prefrontal Cortex of Mice." Neuroscience 432 (April): 44-54. https://doi.org/10.1016/j.neuroscience.2020.02.013.

Paul, Jodi R., Jennifer A. Davis, Lacy K. Goode, Bryan K. Becker, Allison Fusilier, Aidan MeadorWoodruff, and Karen L. Gamble. 2020. "Circadian Regulation of Membrane Physiology in Neural Oscillators throughout the Brain." European Journal of Neuroscience 51 (1): 109-38. https://doi.org/10.1111/ejn.14343.

Pena-Bravo, Jose Ignacio, Rachel Penrod, Carmela M. Reichel, and Antonieta Lavin. 2019. "Methamphetamine Self-Administration Elicits Sex-Related Changes in Postsynaptic Glutamate Transmission in the Prefrontal Cortex." ENeuro 6 (1): ENEURO.0401-18.2018. https://doi.org/10.1523/ENEURO.0401-18.2018.

Perry, Christina J., Erin J. Campbell, Katherine D. Drummond, Jeremy S. Lum, and Jee Hyun Kim. 2021. "Sex Differences in the Neurochemistry of Frontal Cortex: Impact of Early Life Stress." Journal of Neurochemistry 157 (4): 963-81. https://doi.org/10.1111/jnc.15208. 
634 Petrie, Kimberly A., Michael Bubser, Cheryl D. Casey, M. Duff Davis, Bryan L. Roth, and Ariel Y. Deutch. 2004. "The Neurotensin Agonist PD149163 Increases Fos Expression in the Prefrontal Cortex of the Rat." Neuropsychopharmacology 29 (10): 1878-88. https://doi.org/10.1038/sj.npp.1300494.

Piette, Charlotte, Marie Vandecasteele, Clémentine Bosch-Bouju, Valérie Goubard, Vincent Paillé, Yihui Cui, Alexandre Mendes, et al. 2021. “Intracellular Properties of Deep-Layer Pyramidal Neurons in Frontal Eye Field of Macaque Monkeys." Frontiers in Synaptic Neuroscience 13: 725880. https://doi.org/10.3389/fnsyn.2021.725880.

Popoli, Maurizio, Zhen Yan, Bruce S. McEwen, and Gerard Sanacora. 2012. "The Stressed Synapse: The Impact of Stress and Glucocorticoids on Glutamate Transmission." Nature Reviews Neuroscience 13 (1): 22-37. https://doi.org/10.1038/nrn3138.

Radnikow, Gabriele, and Dirk Feldmeyer. 2018. "Layer- and Cell Type-Specific Modulation of Excitatory Neuronal Activity in the Neocortex." Frontiers in Neuroanatomy 12. https://doi.org/10.3389/fnana.2018.00001.

Saffari, R., Z. Teng, M. Zhang, M. Kravchenko, C. Hohoff, O. Ambrée, and W. Zhang. 2016. "NPY+-, but Not PV+- GABAergic Neurons Mediated Long-Range Inhibition from Infra- to Prelimbic Cortex." Translational Psychiatry 6 (February): e736. https://doi.org/10.1038/tp.2016.7.

Saitoh, Akiyoshi, Masanori Ohashi, Satoshi Suzuki, Mai Tsukagoshi, Azusa Sugiyama, Misa Yamada, Jun-Ichiro Oka, Masatoshi Inagaki, and Mitsuhiko Yamada. 2014. "Activation of the Prelimbic Medial Prefrontal Cortex Induces Anxiety-like Behaviors via N-Methyl-D-Aspartate ReceptorMediated Glutamatergic Neurotransmission in Mice." Journal of Neuroscience Research 92 (8): 1044-53. https://doi.org/10.1002/jnr.23391.

Sato, Keisaku, Fanyin Meng, Heather Francis, Nan Wu, Lixian Chen, Lindsey Kennedy, Tianhao Zhou, et al. 2020. "Melatonin and Circadian Rhythms in Liver Diseases: Functional Roles and Potential Therapies." Journal of Pineal Research 68 (3): e12639. https://doi.org/10.1111/jpi.12639. 
660 Shansky, Rebecca M., C. Glavis-Bloom, D. Lerman, P. McRae, C. Benson, K. Miller, L. Cosand, Thomas L. Horvath, and A. F. T. Arnsten. 2004. "Estrogen Mediates Sex Differences in StressInduced Prefrontal Cortex Dysfunction." Molecular Psychiatry 9 (5): 531-38. https://doi.org/10.1038/sj.mp.4001435.

Shansky, Rebecca M., and Anne Z. Murphy. 2021. "Considering Sex as a Biological Variable Will Require a Global Shift in Science Culture." Nature Neuroscience 24 (4): 457-64. https://doi.org/10.1038/s41593-021-00806-8.

Sotres-Bayon, Francisco, Christopher K. Cain, and Joseph E. LeDoux. 2006. "Brain Mechanisms of Fear Extinction: Historical Perspectives on the Contribution of Prefrontal Cortex." Biological Psychiatry 60 (4): 329-36. https://doi.org/10.1016/j.biopsych.2005.10.012.

Ting, Jonathan T., Brian R. Lee, Peter Chong, Gilberto Soler-Llavina, Charles Cobbs, Christof Koch, Hongkui Zeng, and Ed Lein. 2018. "Preparation of Acute Brain Slices Using an Optimized NMethyl-D-Glucamine Protective Recovery Method." JoVE (Journal of Visualized Experiments), no. 132 (February): e53825. https://doi.org/10.3791/53825.

Velasco, Ezequiel Marron Fernandez de, Matthew Hearing, Zhilian Xia, Nicole C. Victoria, Rafael Luján, and Kevin Wickman. 2015. "Sex Differences in GABABR-GIRK Signaling in Layer 5/6 Pyramidal Neurons of the Mouse Prelimbic Cortex." Neuropharmacology 95 (August): 353-60. https://doi.org/10.1016/j.neuropharm.2015.03.029.

Vertes, Robert P. 2006. “Interactions among the Medial Prefrontal Cortex, Hippocampus and Midline Thalamus in Emotional and Cognitive Processing in the Rat." Neuroscience 142 (1): 1-20. https://doi.org/10.1016/j.neuroscience.2006.06.027.

Wallace, Naomi K., Felicity Pollard, Marina Savenkova, and Ilia N. Karatsoreos. 2020. "Effect of Aging on Daily Rhythms of Lactate Metabolism in the Medial Prefrontal Cortex of Male Mice." Neuroscience 448 (November): 300-310. https://doi.org/10.1016/j.neuroscience.2020.07.032.

Weaver, David R. 1998. “The Suprachiasmatic Nucleus: A 25-Year Retrospective.” Journal of Biological Rhythms 13 (2): 100-112. https://doi.org/10.1177/074873098128999952. 
686 Woodruff, Elizabeth R., Lauren E. Chun, Laura R. Hinds, Nicholas M. Varra, Daniel Tirado, Sarah J. Morton, Colleen A. McClung, and Robert L. Spencer. 2018. "Coordination between Prefrontal Cortex Clock Gene Expression and Corticosterone Contributes to Enhanced Conditioned Fear Extinction Recall." ENeuro 5 (6). https://doi.org/10.1523/ENEURO.0455-18.2018.

Workman, E. R., P. C. G. Haddick, K. Bush, G. A. Dilly, F. Niere, B. V. Zemelman, and K. F. RaabGraham. 2015. "Rapid Antidepressants Stimulate the Decoupling of GABA(B) Receptors from GIRK/Kir3 Channels through Increased Protein Stability of 14-3-3n." Molecular Psychiatry 20 (3): 298-310. https://doi.org/10.1038/mp.2014.165.

Xu, Pan, Ai Chen, Yipeng Li, Xuezhi Xing, and Hui Lu. 2019. "Medial Prefrontal Cortex in Neurological Diseases." Physiological Genomics 51 (9): 432-42. https://doi.org/10.1152/physiolgenomics.00006.2019.

Yan, Jie, Jing-Cheng Li, Mei-Lan Xie, Dan Zhang, Ai-Ping Qi, Bo Hu, Wei Huang, Jian-Xia Xia, and ZhiAn Hu. 2011. "Short-Term Sleep Deprivation Increases Intrinsic Excitability of Prefrontal Cortical Neurons." Brain Research 1401 (July): 52-58. https://doi.org/10.1016/j.brainres.2011.05.032.

Yaniv, Yael, and Edward G. Lakatta. 2015. “The End Effector of Circadian Heart Rate Variation: The Sinoatrial Node Pacemaker Cell." BMB Reports 48 (12): 677-84. https://doi.org/10.5483/BMBRep.2015.48.12.061.

Yuen, Eunice Y., Wenhua Liu, Ilia N. Karatsoreos, Jian Feng, Bruce S. McEwen, and Zhen Yan. 2009. “Acute Stress Enhances Glutamatergic Transmission in Prefrontal Cortex and Facilitates Working Memory." Proceedings of the National Academy of Sciences 106 (33): 14075-79. https://doi.org/10.1073/pnas.0906791106.

Yuen, Eunice Y., Jing Wei, and Zhen Yan. 2016. "Estrogen in Prefrontal Cortex Blocks Stress-Induced Cognitive Impairments in Female Rats." The Journal of Steroid Biochemistry and Molecular Biology 160 (June): 221-26. https://doi.org/10.1016/j.jsbmb.2015.08.028.

Zaitsev, A. V., N. V. Povysheva, G. Gonzalez-Burgos, and D. A. Lewis. 2012. "Electrophysiological Classes of Layer 2/3 Pyramidal Cells in Monkey Prefrontal Cortex." Journal of Neurophysiology 108 (2): 595-609. https://doi.org/10.1152/jn.00859.2011. 
A

ZT 0-4

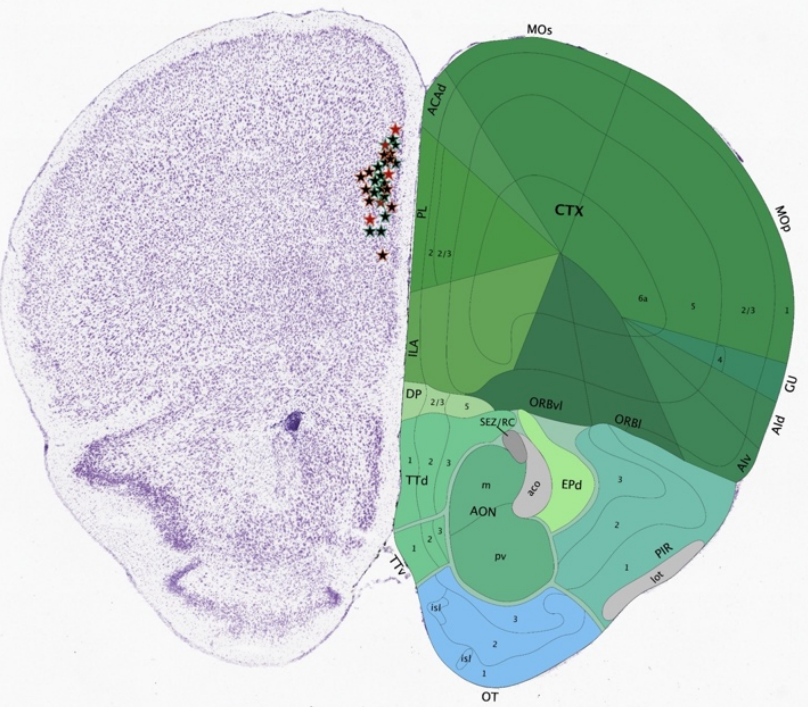

C

ZT 12-16

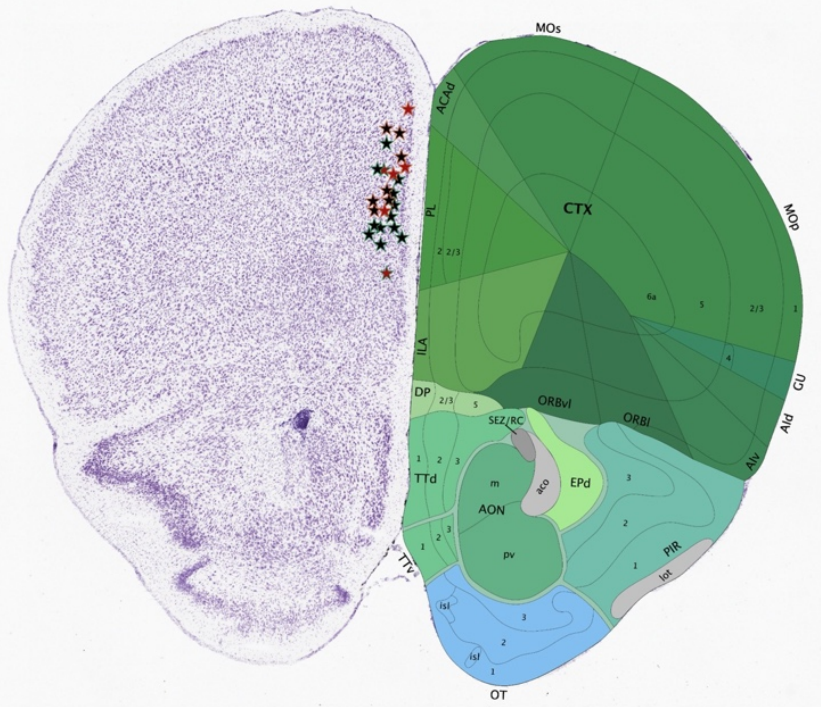

B

ZT 6-10

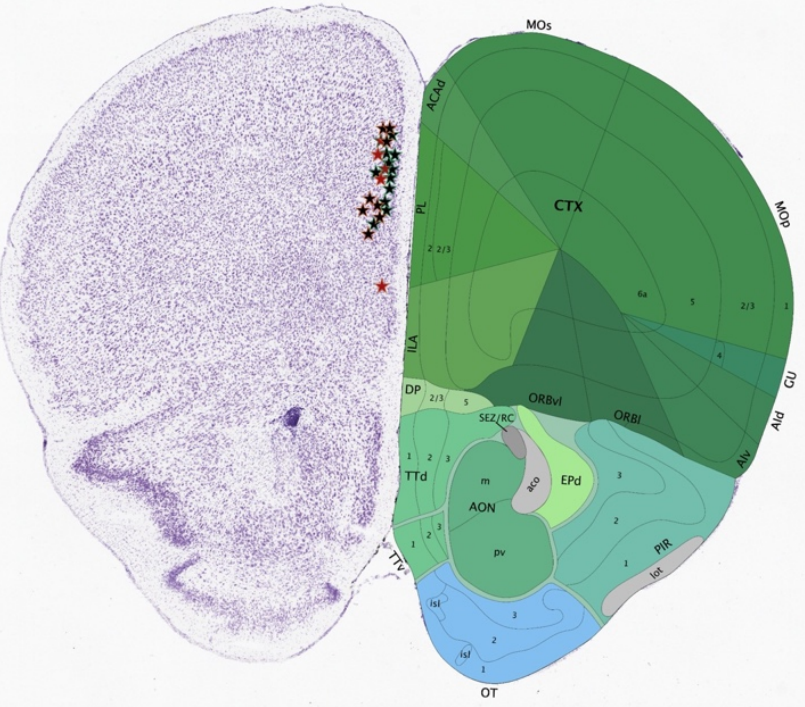

D
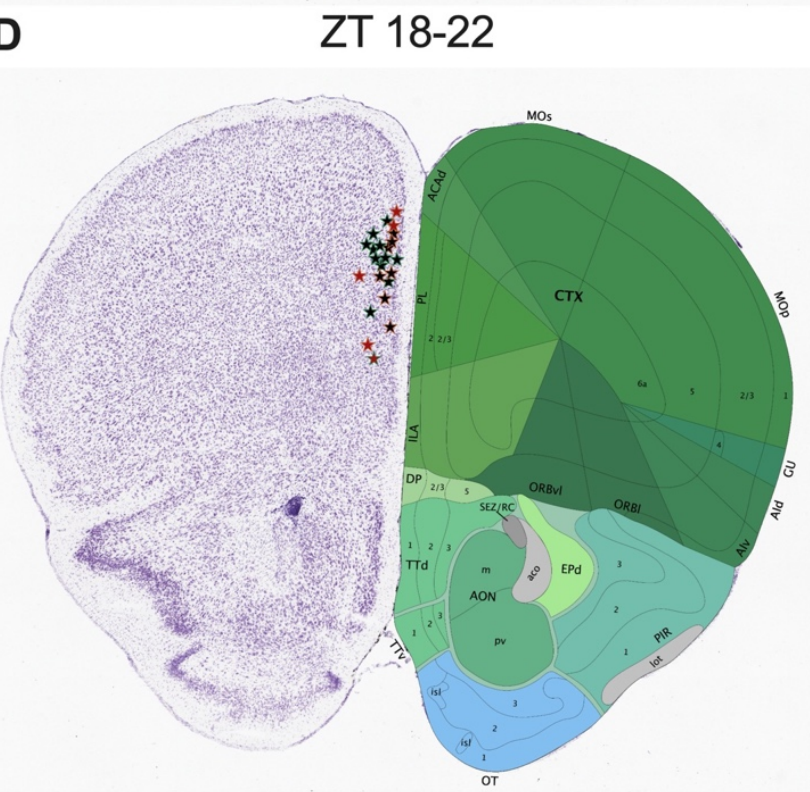

Figure 1 - figure supplement 1. Recording map for layer 2/3 pIPFC pyramidal neurons.

Coronal sections of forebrain showing individual recording sites from majority of neurons that were imaged at (A) ZT0-4, (B) 6-10, (C) 12-16 and (D) 18-22 for basal membrane property, sEPSC, and evoked action potential experiments in male (bluish green outline) and female (vermillian outline) mice. Stars filled with black represent 'Type I' neurons included for analysis and red stars represent Type II/III neurons excluded from analysis. 
A
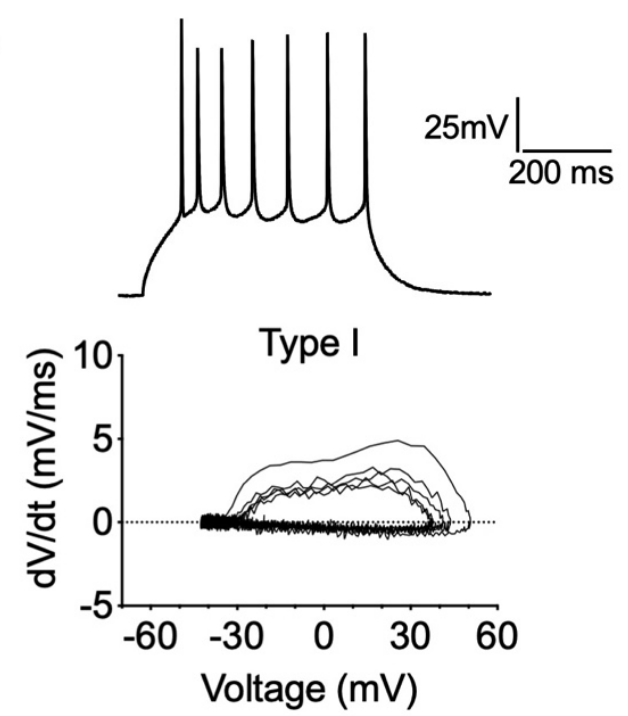

B

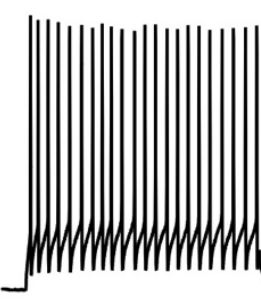

Type II

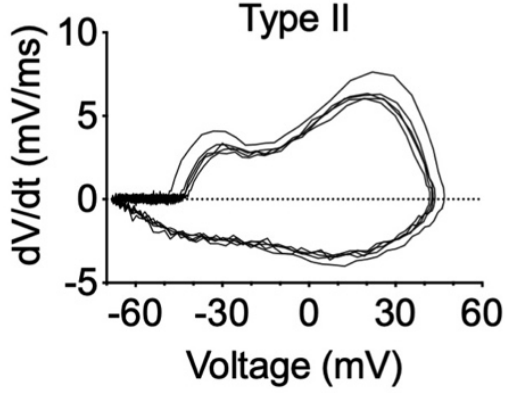

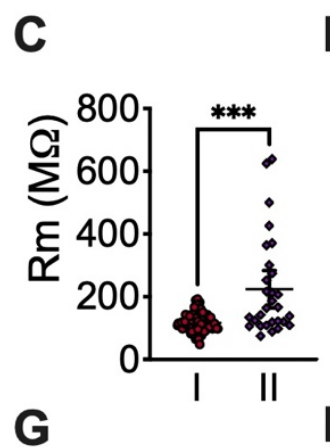

G

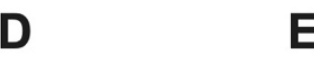

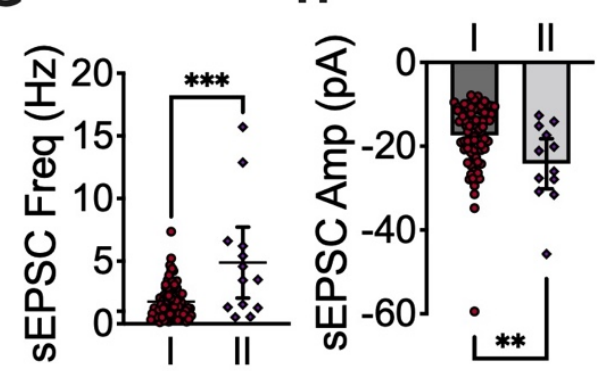

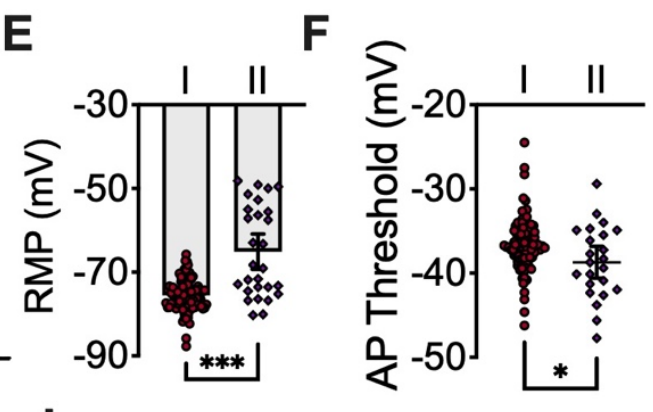

I

Percentage of Cells

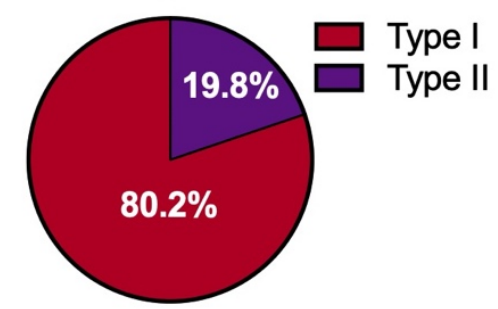

Figure 1 - figure supplement 2. Categories and distinct physiological characteristics of pIPFC

neurons. (A) Representative evoked action potential traces and phase plot diagram of first five action potentials (bottom) illustrating differences in velocity, trajectory, and amplitude in Type I and (B) Type II neurons. (C) Comparison of membrane resistance (Rm), (D) membrane capacitance $(\mathrm{Cm}),(\mathrm{E})$ resting membrane potential $(\mathrm{RMP}),(\mathrm{F})$ action potential $(\mathrm{AP})$ threshold, (G) sEPSC frequency (Freq), and (H) sEPSC amplitude (Amp). (I) Percentage of recorded cells displaying Type I or Type II characteristics (combined among all ZT bins; calculated by $n$ values from AP threshold ). Error bars represent $\pm 95 \% \mathrm{Cl}$. Unpaired student t-test, ${ }^{*} p<0.05,{ }^{* *} p<$ $0.01,{ }^{* * *} p<0.001$. Exact $p$-values and analysis in Figure 1 - supplemental source data 1 . 